\title{
Propuesta para vivienda emergente con perspectiva ZIH en Guerrero, México
}

\author{
Azucena Mendoza ${ }^{1}$ | Claudia Muñoz ${ }^{2}$ | Gerardo Saelzer ${ }^{3}$ | Carlos Carrazco 4 \\ Recibido: 08-01-2020 | Versión final: 02-07-2021
}

Resumen El objetivo de esta investigación es analizar el estado de neutralidad de los componentes de una vivienda de emergencia en México, desarrollada con recursos públicos, a través de las emisiones de $\mathrm{CO}_{2}$ generadas en su uso. Se muestran los resultados de un estudio comparativo de las viviendas de emergencia, en tres localidades del Estado de Guerrero, México, y propone recomendaciones para construirlas bajo el concepto de "vivienda de bajo impacto". Se empleó el concepto ZIH (Zero Impact Housing - Vivienda de Impacto Cero) adaptado a la etapa de uso, que considera variables como Suelo, Agua y Energía. Los temas también fueron abordados bajo un nuevo enfoque de sustentabilidad asociado a cuatro pilares; gubernamental, social, económico y medio ambiental. La investigación permitió generar recomendaciones para el desarrollo de vivienda de emergencia en México, integrando elementos que incrementan de la habitabilidad hasta en un 42\% y la disminución de costos de energía anuales hasta $\$ 28,772.60$. Se espera que la presente metodología sirva de referencia para futuros análisis en otras regiones del país o de Latinoamérica. La actual política de vivienda busca atender a la población más vulnerable de México, pero carece de consideraciones sobre el uso sustentable de los recursos, la habitabilidad y el impacto al medio ambiente; la presente investigación genera las propuestas con criterios de sustentabilidad para integrar dichos aspectos sumando a los esfuerzos vigentes, bajo una perspectiva de desarrollo sustentable.

Palabras clave: Indicadores ZIH; desastres naturales; gobierno federal

Citación

Mendoza, A. et al. (2021). Propuesta para vivienda emergente con perspectiva ZIH en Guerrero, México. ACE Architecture, City and Environment, 16(47), 9189. DOI: http://dx.doi.org/10.5821/ace.16.47.9189

\section{Proposal for an Emergency Housing Project Under a ZIH Perspective in Guerrero, Mexico}

\begin{abstract}
The This article presents the results of a comparative study of emergency housing, in three locations in the state of Guerrero, Mexico, and proposes recommendations to build them under the concept of "low impact housing". The objective of this research is to analyze the state of neutrality of the components of an emergency housing project in Mexico financed through public resources, from the viewpoint of the $\mathrm{CO}_{2}$ emissions generated from its use, and also analyzing the level of thermal habitability for its occupants. The applied methodology is the $\mathrm{ZIH}$ concept (Zero Impact Housing) adapted to each stage of use, which considers variables such as Soil, Water and Energy. The issues were also addressed under a new sustainability approach associated with four pillars; governmental, social, economic and environmental. This research allowed to generate recommendations for the development of an emergency housing project in Mexico, integrating elements that increase habitability by up to $42 \%$ and decrease annual energy costs by up to $\$ 28,772.60$ pesos. The present methodology is expected to serve as a reference for future analyses in other regions of the country or in Latin America. The current housing policy approach seeks to serve the most vulnerable population in Mexico, but lacks considerations on the sustainable use of resources, habitability and the impact on the environment; This research generates a proposal to include these aspects under a sustainability criterion, adding to the current efforts, under a sustainable development perspective.
\end{abstract}

Keywords: ZIH indicators; natural disasters; federal government

${ }^{1}$ M.Sc. Arquitecta, Facultad de Arquitectura, Construcción y Diseño, Universidad del Bío-Bío, Chile (ORCiD: 0000-0002-5342-7679), ${ }^{2}$ Dra. en Arquitectura y Urbanismo, Departamento Ciencias de la Construcción, Universidad del Bío-Bío, Chile (ORCiD: 0000-0002-4859-478X, WoS ResearcherID: K-5375-2014), Departamento de Diseño y Teoría de la Arquitectura, Universidad del Bío-Bío, Chile (ORCiD: 0000-00024289-8379), ${ }^{4}$ M. Eng. Arquitecto, Instituto Tecnológico Autónomo de México, ITAM - Universidad Politécnica de Madrid, UPM (ORCiD: 0000-0003-1986-2949). Correo de contacto: azucena mendozal@hotmail.com 


\section{Introducción}

Los fenómenos naturales son parte de nuestro medio ambiente natural, si nos adaptamos nos permiten convivir con ellos. Cuando es referida a una catástrofe por una manifestación de estos eventos naturales es porque son con magnitud e impacto considerables, ocasionando pérdidas materiales y humanas debido a condiciones de vulnerabilidad socioeconómicas y físicas.

De acuerdo a United Nations Office for Disaster Risk Reduction (UNISDR), los desastres no son naturales, sino que son el resultado de las omisiones y la falta de prevención y planificación ante los fenómenos de la naturaleza, es decir, que el fenómeno es natural, sin embargo, que el desastre es por la acción del hombre en su entorno (The World Bank and The United Nations 2010).

En México existen diferentes tipos de catástrofes ocasionadas por fenómenos meteorológicos, entre los que destacan los fenómenos geológicos e hidrometeorológicos y dentro de ellos se encuentran los huracanes, (que comprenden tormentas e inundaciones) y terremotos; cada uno considera un $27 \%$ de la población total, así como el $41 \%$ y 27\% de superficie del territorio nacional (The World Bank and The United Nations 2010).

Los desastres son medidos por los impactos socio - económicos y por los daños y pérdidas generados en la población; incluyendo el número de heridos y decesos, infraestructura y edificación dañada. El impacto total de ellos dependerá de la envergadura, magnitud y el número anual de fenómenos naturales en la República Mexicana.

Desde el terremoto de 1985, México inició una actualización normativa y política en materia de atención a las afectaciones que los desastres naturales ocasionan a la población. Dichas consideraciones incluyen la previsión, la atención durante y la actuación postdesastre. Como una de las principales modificaciones a la estructura institucional, se plantea la creación del Fideicomiso Fondo de Desastres Naturales (FONDEN), que tiene por objeto proporcionar auxilio y ayuda a la población que se encuentra ante la inminencia de un fenómeno natural poniendo en riesgo la vida y salud de la sociedad.

De forma paralela, el gobierno mexicano ha generado avances en la política de vivienda buscando disminuir el déficit habitacional, poniendo como premisa que todo mexicano o mexicana tiene derecho a una vivienda digna y decorosa (Diario Oficial de la Federación 2014a), de acuerdo a los derechos humanos y constitucionales.

Esto genera una serie de análisis en cuanto al tipo de soluciones que se requieren implementar, con el objetivo de atender a la población en su totalidad, sin importar su nivel económico, ubicación, edad o condición física. Actualmente, alrededor de 1 millón de soluciones de viviendas se generan al año, de las cuales aproximadamente la mitad son nuevas, (CONAVI, 2018).

Existe un mapa de las instituciones que atienden a cada uno de los sectores de vivienda, estas son: FONDEN (vivienda emergente), CONAVI (define la política de vivienda y maneja el programa de subsidio con esquemas financieros para la vivienda) y la Sociedad Hipotecaria Federal (es la banca de desarrollo). Debido a esto surge la necesidad de fortalecer la coordinación interinstitucional, para asegurar que las agendas particulares fortalezcan la visión general que tiene el país, aplicando un programa integral de vivienda asequible, digna y sustentable para todos, tal como se estipula en el Programa Nacional de Vivienda 2014-2018 (Diario Oficial de la Federación, 2014b). 
Debemos mencionar que, dada tal necesidad de coordinación, mientras en algunas ramas de la vivienda (particularmente la de construcción en serie), se han dado grandes avances en materia de sustentabilidad, evaluación del impacto ambiental, eficiencia energética y confort, existen también otras agendas en las cuales estos conceptos no han podido ser incorporados, como el caso de la vivienda emergente, tema de esta investigación.

Tomando en cuenta lo anterior, se generan las siguientes interrogantes: ¿En qué condiciones se encuentra la vivienda de emergencia FONDEN, vista desde la perspectiva federal de vivienda sustentable mexicana?, ¿tienen características sustentables?, ¿se somete la vivienda de emergencia FONDEN a una evaluación de impacto cero durante la etapa de uso?, ¿su diseño y construcción integran la aceptación social?, ¿qué grado de compatibilidad tendría desarrollar una propuesta de vivienda de bajo impacto, con las actuales directrices de la política de vivienda sustentable mexicana?.

Para responder a estas interrogantes, se considera como objetivo analizar el estado de neutralidad de los componentes de una vivienda de emergencia en México, construida con recursos de FONDEN, considerando las emisiones de $\mathrm{CO}_{2}$ generadas en durante su uso, examinando el nivel de habitabilidad térmica de los ocupantes, para proponer recomendaciones que se puedan llevar a la práctica bajo un concepto de "Vivienda de Bajo Impacto".

Se debe dejar constancia si dentro del diseño y construcción se considera una respuesta del grado de factibilidad para los usuarios, agregando el nivel de impacto que estas generarían al estar ocupadas.

En esta investigación se aborda el concepto de sustentabilidad examinando cuatro pilares: el gubernamental, el social, el económico y el medio ambiental, así mismo, aplicamos dos criterios para la evaluación de la vivienda sustentable:

1. Para la selección del caso de estudio se eligió el Estado de Guerrero, al ser el más vulnerable de todo el territorio nacional, ya que cuenta con el mayor número de tipologías de fenómenos naturales y ocupa desde el año 2000, uno de los primeros tres lugares a nivel nacional en índice de rezago social (CONEVAL 2016).

2. Mediante un análisis tipológico se genera el caso base de vivienda de emergencia FONDEN y se considera como localidad base el desarrollo Nuevo Azinyahualco, cercano a la capital del estado, Chilpancingo de los Bravo (clima templado) y, además, el mismo caso se emplaza en dos de los climas extremos que tiene el Estado; cálido, en la localidad de Ixtapilla y semifrío, en San Vicente.

Este modelo es el que sirve como estructura para el desarrollo de la evaluación ZIH (Zero Impact Housing - Vivienda de impacto cero) de la propuesta de vivienda de emergencia.

La investigación ofrece recomendaciones para desarrollar la vivienda emergente en México, con una visión amplia de sustentabilidad y una restructuración en la metodología de ZIH (Figura 1), al ser considerada en el ciclo de vida solo en la etapa de uso.

Esto anterior, de acuerdo con la actual política de vivienda sustentable, en donde se hacen consideraciones en áreas de: Habitabilidad térmica, energía, agua y suelo. 
Figura 1. Mapa conceptual del sistema de evaluación ZIH propuesto para la vivienda de emergencia FONDEN bajo una perspectiva de desarrollo sustentable

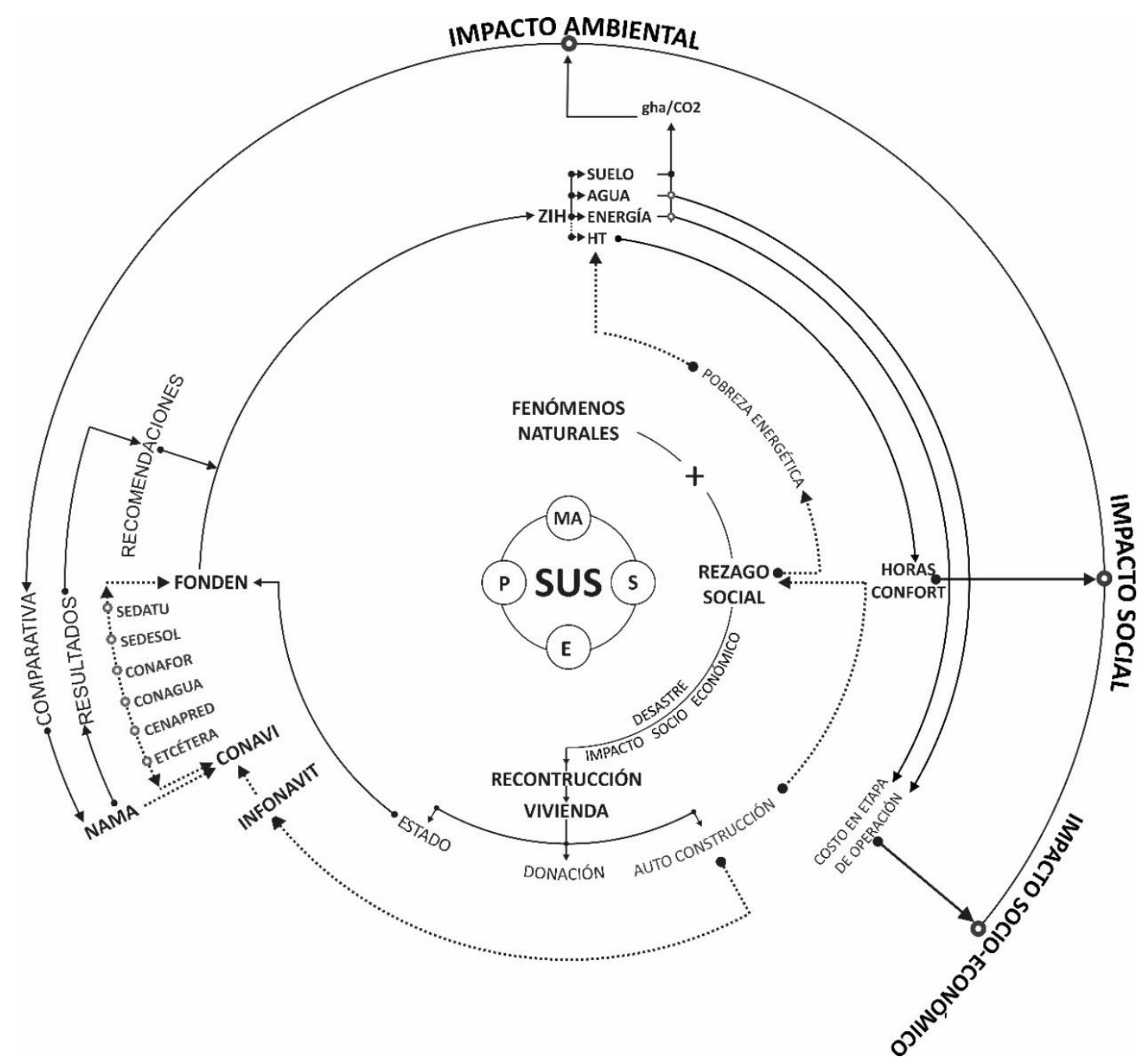

Fuente: Elaboración propia.

\section{Impacto socioeconómico}

En el Estado de Guerrero se registran fenómenos naturales que se encuentran dentro de dos categorías: fenómenos geológicos e hidrometereológicos. Se dan otros fenómenos originados por la intervención del hombre, como los fenómenos químicos, socio-organizativos y sanitarios. En lo social, el Estado cuenta con una población indígena que asciende al 15\% del total (INEGI 2010), que viven en su mayoría en forma marginal en la Sierra; un 69.7\% (Consejo Nacional de Evaluación de la Politica de Desarrollo Social., 2013) del total de la población de Guerrero está en pobreza, el Estado tiene el primer lugar del país en carencia por calidad y espacios de la vivienda con $33.4 \%$ y, el segundo lugar en carencia de acceso a los servicios básicos en la vivienda con un $59.4 \%$ del total de la población (CONEVAL, 2016), todo lo anterior aumenta la vulnerabilidad social ante la aparición de un fenómeno natural de alta envergadura. 
Entre las diferentes variables que involucran rezago social está la vivienda. En Guerrero el 13.88\% de la población tienen pisos de tierra, el 12.98\% no dispone sanitario, el 15.14\% no dispone agua entubada de la red pública, el 16.28\% no dispone drenaje y el $2.48 \%$ no dispone energía eléctrica (CONEVAL, 2016). Esta estadística hace que el rezago social en el Estado sea de un grado "Muy alto" desde el año 2000, ubicándose en los primeros tres lugares, y al año 2015 ocupe el segundo lugar a nivel nacional, seguido de Oaxaca.

Los acontecimientos naturales que existen en la zona, unidos al rezago social, fortalecen los desastres de origen natural, teniendo del 2000 al 2018, 29 declaratorias de desastre, 26 declaratorias de emergencia y 9 contingencias climatológicas (CENAPRED, 2018b).

En un año pueden existir diversos tipos de fenómenos naturales que azotarán la zona, sin embargo, algunos causan mayores estragos en la vivienda de la zona como se observa en la Figura 2. Estos deberán superar la capacidad del Estado para que actúe FONDEN, donde algunos fenómenos que azotan un lugar llegan a tener declaratoria (actuando FONDEN), y otros fenómenos que también causan estragos en la zona los asume el Estado (Tabla 1).

Figura 2. Viviendas dañadas por tipo de fenómeno en Guerrero del 2010-2018

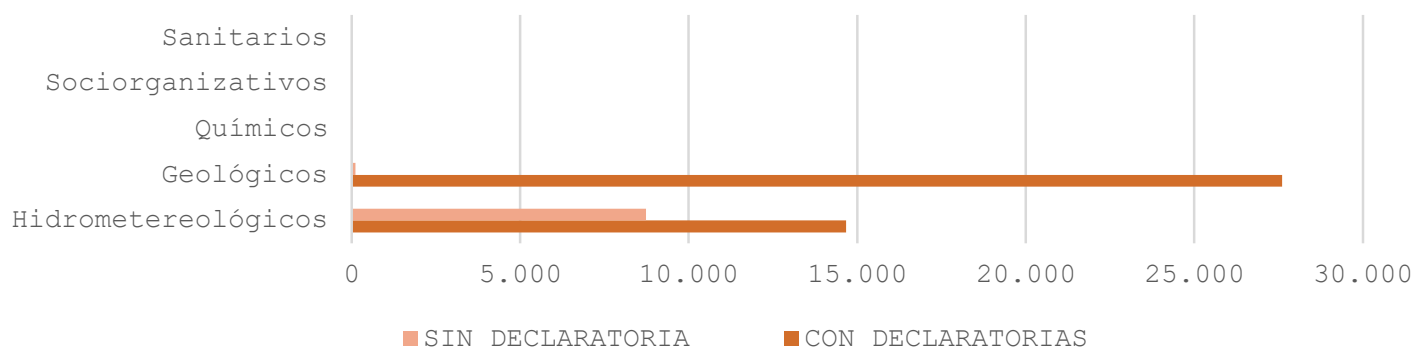

Fuente: Elaboración propia con datos de resumen de impacto socioeconómico desastres 2000-2015 de CENAPRED 2018b.

Tabla 1. Resumen de los impactos socioeconómicos de Guerrero del año 2010 al 2016

\begin{tabular}{|c|c|c|c|c|c|c|c|c|}
\hline \multirow{2}{*}{ AÑO } & \multicolumn{4}{|c|}{ CON DECLARATORIAS } & \multicolumn{4}{c|}{ SIN DECLARATORIA } \\
\cline { 2 - 10 } & Muertes & $\begin{array}{c}\text { Casos de } \\
\text { afectación }\end{array}$ & $\begin{array}{c}\text { Viviendas } \\
\text { dañadas }\end{array}$ & $\begin{array}{c}\text { Total de } \\
\text { daños (MDP) }\end{array}$ & Muertes & $\begin{array}{c}\text { Casos de } \\
\text { afectación }\end{array}$ & $\begin{array}{c}\text { Viviendas } \\
\text { dañadas }\end{array}$ & $\begin{array}{c}\text { Total de } \\
\text { daños (MDP) }\end{array}$ \\
\hline 2010 & 0 & 6200 & 467 & 108,51 & 36 & 33.401 & 5.282 & 59,45 \\
\hline 2011 & 2 & 3400 & 654 & 35,07 & 26 & 5.061 & 934 & 21,95 \\
\hline 2012 & 4 & 187.087 & 20.836 & 976,3 & 42 & 4.369 & 632 & 21,08 \\
\hline 2013 & 105 & 281.738 & 10.592 & $23.684,43$ & 6 & 2.768 & 286 & 44 \\
\hline 2014 & 8 & 54.954 & 8.914 & 783,42 & 17 & 7.298 & 1.402 & 20,36 \\
\hline 2015 & 3 & 139.440 & 695 & 470,7 & 14 & 1.913 & 321 & 10,19 \\
\hline 2016 & 2 & 411.344 & 122 & $1.969,80$ & & & & 177,02 \\
\hline TOTAL & 124 & 1.084 .163 & 42.280 & $28.028,22$ & 141 & 54.810 & 8.857 & 1702 \\
\hline
\end{tabular}

Fuente: Elaboración propia con datos obtenidos de la serie "Impacto socioeconómico en México, tomo 12 al 18 ". Y resumen de impacto socioeconómico desastres 2000 - 2015 de CENAPRED 2018b. * En el total de los daños se considera la vivienda y otros sectores.

La Tabla 1 muestra, en resumen, los impactos sufridos en Guerrero desde el año 2010 al 2016, solo considerándose defunciones, casos de afectación población afectada y vivienda, aunque un desastre natural, considera otras dimensiones físicas y ambientales afectadas. En el total de daños se consideran los daños generados a escuelas, unidades de salud, área de cultivo y unidades 
económicas. El presupuesto que entrega FONDEN para la construcción de una vivienda es de $\$ 120,000.00$ MXN y, cuando es reubicación, se agrega el terreno y, para que la vivienda funcione, tienen que estar activos todos los demás sectores que sufrieron daño por el acontecimiento. La vivienda no se considera aisladamente, forma parte en un contexto urbano y debe relacionarse con los demás sectores para acceder a los recursos FONDEN. Aunque la investigación está centrada en una vivienda aislada, debemos señalar que FONDEN del año 2010 al 2016 otorgó presupuesto para 42,280 viviendas, con un total de $\$ 28,028.22$ millones de pesos mexicanos, esta estadística cubre solo las que tuvieron declaratoria en el Estado de Guerrero.

\section{Análisis tipológico de las viviendas emergentes}

Para el análisis de casos que se distribuyen dentro de territorio nacional mexicano, se desglosan las tres categorías; vivienda de emergencia, vivienda progresiva y vivienda digna.

- La vivienda de emergencia está planeada para que se ocupe en un lapso determinado, mientras se concreta su situación definitiva. Como su nombre lo dice, nace de un incidente perturbador de la vida cotidiana, en que el usuario damnificado se queda sin cobijo y se requiere una atención inmediata multisectorial: se debe concebir con una planeación del proyecto habitacional antes de la contingencia y un cuidado psicológico del usuario al momento de la intervención arquitectónica. Algunos de los constructores de esta tipología provienen de la ONG, y se caracterizan por destacar la planeación para llevar a cabo la realización de una vivienda dentro de un estado de contingencia. El sistema constructivo que se aborda en cada una de las edificaciones varía, puede ser de fácil ensamblado o vernacular, y consideran en su mayoría a la población afectada durante la fase de construcción. Debido a que consideran la planeación de la fase constructiva y el análisis de aceptación del damnificado, acotan el tiempo de construcción de la vivienda.

- La vivienda progresiva permite un aumento o crecimiento de forma paulatina; es necesario partir con una solución habitacional, que no sea de carácter definitivo, pero que les permita una evolución y flexibilidad en su construcción, es decir, "soluciones inicialmente modestas pero mejorables en el tiempo" (Mac Donald, 1987); esto conlleva a que, para que el usuario pueda hacer uso de una solución modesta, ésta debe contener las condiciones mínimas de habitabilidad para que pueda subsistir decorosamente e ir mejorando con el tiempo.

- Vivienda definitiva. Según el artículo 2, de la Ley de Vivienda del Diario Oficial de la Federación el 27 de junio del 2006 dice: "Se considerará vivienda digna y decorosa la que cumpla con las disposiciones jurídicas aplicables en materia de asentamientos humanos y construcción, habitabilidad, salubridad, cuente con los servicios básicos y brinde a sus ocupantes seguridad jurídica en cuanto a su propiedad o legítima posesión, y contemple criterios para la prevención de desastres y la protección física de sus ocupantes ante los elementos naturales potencialmente agresivos“(Diario Oficial de la Federación, 2014a). En cuanto a la ONU dice que como mínimo debe reunir siete criterios (Naciones Unidas - Derechos Humanos - ONU HABITAT, 1948), que son: la seguridad de la tenencia, disponibilidad de servicios, materiales, instalaciones e infraestructura, asequibilidad, habitabilidad, accesibilidad, ubicación y adecuación cultural.

La vivienda FONDEN en el Estado de Guerrero, originada por un incidente perturbador, tiene que ser construida en un acotado lapso y con la intención de ser definitiva. 


\section{Alcances de la vivienda impacto cero (Zero Impact Housing - ZIH) en etapa de operación}

En una medición conjunta de los diferentes factores que intervienen en las emisiones de $\mathrm{CO}_{2}$, se opta por la propuesta de Shady Attia y André De Herde en la definición de edificios de Impacto cero. Ellos analizan cuatro factores de manera conjunta y relacionándolos entre sí, estos son: agua, energía, materiales y uso de la tierra. Enfatizan que uno de los problemas más comunes sucede cuando se trata cada recurso por separado, porque la realidad de los edificios y sus ciclos de vida es que son dinámicos (Attia y De Herde, 2011). Esta propuesta contempla el análisis de una vivienda durante todo su ciclo de vida en cada uno de los parámetros.

Debido a que la propuesta solo se aplica durante la etapa de operación, se ajustaron algunas variables acordes al objetivo general, se consideraron tres parámetros base; el diseño, los artefactos fijos y los materiales. Una combinación de materiales y el diseño de la edificación nos dará la habitabilidad térmica, es decir, horas - confort - año, de los recintos evaluados. En caso de no encontrarse dentro de la zona de confort, se analiza con sistemas activos de climatización y con ello se obtiene la demanda energética, el costo energético y las emisiones de $\mathrm{CO}_{2}$ que genera la implementación del sistema. El consumo de agua también varía dependiendo los artefactos y el diseño implementado, esto deberá arrojar resultados, tanto en consumo de agua como en las emisiones que ellas generarían. Aunando estos dos elementos se define cuantas emisiones de $\mathrm{CO}_{2}$ genera la vivienda por $\mathrm{m}^{2}$.

\subsection{Análisis de habitabilidad térmica (HT)}

La habitabilidad como un concepto amplio aplicado a la arquitectura abarca desde la percepción (físico espacial / psicosocial), el confort (acústico / térmico / lumínico) y la seguridad (higiene / fuego y accidentes); su intento es definir las características mínimas con las que debería contar una vivienda para lograr el bienestar del usuario. Considerando la estructura y materialidad, usos y mantención, contar con la percepción, confort y seguridad, para satisfacer las necesidades de los habitantes ( D’Alençon, et al, 2008).

Todos los diseños arquitectónicos deberían estar fundados sobre los parámetros de habitabilidad, para la propuesta de vivienda de impacto cero no solo debe ser necesario analizar energía, si no también analizar las condiciones de confort térmico que los usuarios tendrán al hacer uso de la vivienda; por ello se opta por el análisis de habitabilidad térmica, que es "la relación de las horas en que la vivienda entrega las condiciones de bienestar térmico requerido en relación a las horas totales del período de estudio" (Sarmiento \& Hormazábal, 2016). Un factor determinante en la habitabilidad térmica $(\mathrm{HT})$ es definir un rango de confort con los que serán evaluados todos los recintos de la vivienda.

\subsection{Energía}

La energía es un recurso que se encontrará dentro de todas las etapas del ciclo de vida de la vivienda, para considerarla solo en el uso, su efectividad y eficiencia dependerá en gran medida del compromiso y disponibilidad de todos los actores que se encuentran involucrados (Dalgleish et al., 2007) jugando un papel principal, la cultura local. Ahora, si se considerara todo el edificio, y se agrega energía renovable y almacenamiento de ella, se tienen reducciones significativas (Jones et al, 2017), pero una variable en el uso de este tipo de tecnologías es su alto costo (Attia, 2011) que podría generar una desventaja al momento de adquirirlas. 
En el potencial de la eficiencia deben considerarse factores que influyen en la demanda energética, como; los parámetros climáticos, la envolvente del edificio, sistemas pasivos (Pardo \& Thiel, 2012), ocupantes, sistemas de energía (R. Džiugait 'e-Tum 'enien 'e, V. Jankauskas, 2015), y a partir de aquí dar soluciones constructivas para mejorar el rendimiento durante la fase de operación, sin embargo, al hacer esto, es posible que se pueda desfasar el impacto a la fase de construcción, ya que los materiales usados para mayor aislamiento térmico, no refleja la cantidad de energía incorporada para producir dichos materiales (Valančius et al, 2018). Es por ello que, aunque no se considere la energía incorporada de los materiales, se debe tener presente los materiales orgánicos locales y/o reciclados, o estudios relevantes para no descuidar y atribuir más impactos a otras fases del ciclo de vida de la edificación.

Durante la fase de uso / operación, la demanda energética del edificio dependerá únicamente de lo que ya está, y así, como de las características de los ocupantes. Las emisiones de $\mathrm{CO}_{2}$ generadas por el consumo energético depende del factor de emisión aceptado por SEMARNAT, y aunque, al comparar con otros países podría producir una situación paradójica por que varía según la fuente de producción de energía de cada país (Castellano et al, 2015), se crea una referencia de lo qué está pasando en cada región.

Siguiendo con el fundamento principal del concepto de Impacto cero, "no considerar todos los entes por separado"; se encuentra confusa la metodología adecuada para dar soluciones radicales en la reducción de emisiones de $\mathrm{CO}_{2}$, que también sean duraderas y no perjudiciales (Moran et al, 2014) y además sea viable para las diferentes tipologías de edificación. Con ello nos inclinamos al concepto de que una construcción de impacto cero, debería significar que la eficiencia energética de un edificio se maximiza (Attia, 2011), conservar el "cero" como un punto de partida en las mediciones, que la referencia sea la proximidad que a él se tiene. Para el caso de la vivienda de emergencia de Guerrero, México, se tienen que considerar las diversas variables a nivel país y a nivel región, debido a que diferentes emplazamientos marcan alcances y limitantes.

\subsection{Agua}

Las actividades humanas generan un impacto en los recursos del agua, el cual puede ser medido con la denominada huella hídrica. Existen dos métodos para cuantificar los impactos hídricos, el primero es el Water Footprint Network (WFN) y el segundo es el Análisis de Ciclo de Vida (ACV), enfocado en el impacto (Roibás et al., 2018). El primer modelo fue propuesto originalmente por Hoesktra en el 2002 (Chapagain \& Hoekstra, 2014), quien lo ha catalogado como la cantidad de agua requerida para la producción de bienes y servicios consumidos por los habitantes del país (Lee, 2015; Su, Gao, Guan, \& Su, 2018).

Según el primero, la huella hídrica se clasifica en tres componentes (Ibidhi \& Ben Salem, 2018; Lee, 2015; Roibás et al., 2018):

- La huella hídrica azul: Es la extracción de agua dulce tomada de las escorrentías de agua y/o acuíferos, que no es devuelta a la cuenca original o se devuelve en un lapso diferente.

- La huella hídrica verde: Es el agua superficial que se encuentra en el suelo, y es utilizada por la vegetación (productos agrícolas o bosques).

- La huella hídrica gris: es el agua necesaria para diluir los contaminantes y mantener la calidad de agua cercana.

El segundo método, el impacto basado en ACV (huella hídrica ISO 14046-2014), considera los componentes azules y verdes, pero difiere en el agua gris. Incluye la materia prima a través de la producción, el uso, el tratamiento final de su vida útil, el reciclaje, y la eliminación final (Liang et al., 2018). Los principios de este método se basan en ACV, pero se centra en la disponibilidad y

ACE, 16 (4.7) CC BY-ND 3.0 ES | UPC Barcelona, España | Propuesta para vivienda emergente con perspectiva ZIH en 
degradación del agua, procura considerar los impactos ambientales, mientras que WFN tiene como objetivo la productividad del agua dulce global como un recurso limitado (Pfister et al., 2017).

La presente investigación se centra en el segundo método, solo en la etapa de uso, y hace uso de la herramienta de SAAVI, para obtener un estimado del consumo de agua en litros por persona al día de la vivienda emergente FONDEN.

\subsection{Uso de suelo}

La reducción de las emisiones de $\mathrm{CO}_{2}$ de las construcciones, no depende únicamente de la eficiencia energética de la edificación, sino también con los usos a nivel urbano de la tierra, los servicios, infraestructura, comodidades, áreas verdes con los que cuente el lugar. Algunos autores coinciden que el volumen de los edificios en áreas urbanas, influye vigorosamente en los intercambios térmicos con el entorno urbano, así como el nivel de exposición solar que podría tener (Conticelli et al, 2017), con esto se ve envuelto la planificación a nivel urbano y su densidad.

La planificación urbana densa puede crear un efecto isla de calor (J. Zhang, Xie, Luan, \& Chen, 2015) y reducir el acceso a luz (Conticelli et al., 2017), lo que directamente influye en el consumo energético a nivel residencial, y a su vez, provoca un decaimiento generalizado en la calidad de vida a nivel ciudad. Sin embargo, si la densidad es alta y cuenta con variedad de transportes públicos eficientes, se reduce la ocupación de suelo y se optimiza los recorridos del transporte, reduciendo así la contaminación del aire y deja intactos los suelos de uso forestal o agrícolas (Neuman 2014; Conticelli, Proli y Tondelli 2017).

La deforestación, el colapso por recursos y la acumulación de $\mathrm{CO}_{2}$, están indicando que la demanda puede superar la capacidad de regeneración y absorción de la biosfera (Borucke et al., 2017), por lo que se está implementando en México estrategias de acción temprana (Elizondo et al, 2017) en el que promueven el uso sostenible de los bosques, sistemas agroforestales y silvopastoriles, y además mejoras en la política ambiental incentivando nuevas políticas. Es por ello que para un análisis conjunto de la vivienda y el impacto en el sitio, es necesario llevar a cabo una evaluación de uso de suelo para saber cuánto es la huella ecológica que generaría una población en un lugar establecido por la demanda y actividades que se realicen in situ y la tierra productiva equivalente (Martínez et al, 2017) que es necesaria para producir o absorber estos impactos.

\section{Definición del caso base, vivienda FONDEN}

De acuerdo a la clasificación climática del Estado de Guerrero (Fuentes Freixanet, 2009), este cuenta con tres zonas climáticas divididas en seis climas, pero la vivienda FONDEN en el Estado, tiene la misma tipología en todas sus zonas climáticas. En el año 2013 se produjo un desastre que activó los recursos FONDEN (CENAPRED, 2015) para viviendas en todo el territorio, debemos suponer que las viviendas que se construyeron en esa emergencia fueron de la misma tipología para todos los climas del Estado de Guerrero

La vivienda expuesta en esta investigación es de comunidades rurales, y para la obtención de sus climas y las regiones con más altas y bajas temperaturas se consultó el Atlas Climático Digital de México, Estado de Guerrero (Centro de Ciencias de la Atmósfera UNAM, 2013), estos datos se verificaron con las Normales Climatológicas del SMN (Servicio Metereológico Nacional, 2010) que entregan detalles de los climas, y se verificó el nombre del clima con INEGI (INEGI, 2005), además con archivos EPW se analizaron en el software Climate Consultant. 
En función de lo anteriormente expuesto, los dos climas más críticos del Estado de Guerrero para realizar el estudio de habitabilidad térmica y energía son, Ixtapilla en Cutzamala de Pinzón (clima cálido) y San Vicente de Chilpancingo de Los Bravo (clima semifrío). Como caso base, se considera el clima templado de la comunidad rural "Nuevo Azinyahualco" ubicada en el ejido Zoyatepec, cerca de la capital del Estado, Chilpancingo de los Bravo.

Las temperaturas máximas promedio de Chilpancingo, se encuentran dentro de los meses marzo, abril y mayo, hasta los $34^{\circ} \mathrm{C}$, y en los meses invernales su máxima está en $28^{\circ} \mathrm{C}$. Como mínima en los meses de noviembre, diciembre y enero llega a bajar hasta los $12^{\circ} \mathrm{C}$ y en los meses de verano está en $16-17^{\circ} \mathrm{C}$. La oscilación diaria más baja del año es en el mes de septiembre con $9.8^{\circ} \mathrm{C}$ y la más alta en el mes de marzo con $15.9^{\circ} \mathrm{C}$. La anual está en $12.9^{\circ} \mathrm{C}$. Sus vientos predominantes provienen principalmente del sur - oeste con una velocidad máxima promedio en marzo de $4.5 \mathrm{~m} / \mathrm{s}$.

En Ixtapilla, las temperaturas llegan a casi los $40^{\circ} \mathrm{C}$ en los meses de verano y en los invernales pueden llegar a $31^{\circ} \mathrm{C}$, pero sus temperaturas bajas solo llegan a $24^{\circ} \mathrm{C}$ en verano y $18^{\circ} \mathrm{C}$ en invierno. En San Vicente, la temperatura máxima llega a $27^{\circ} \mathrm{C}$ en los meses de verano y en los meses invernales puede llegar a $23^{\circ} \mathrm{C}$. Las temperaturas mínimas en invierno pueden ser de $2^{\circ} \mathrm{C}$; su temperatura media anual es de $15^{\circ} \mathrm{C}$. La humedad relativa de San vicente es de 25 a $80 \%$ en invierno y a 50 a $90 \%$ en verano, mientras en Ixtapilla es de 40 a $85 \%$ en invierno y 70 a $95 \%$ en verano.

\subsection{Diseño}

Se presenta un diseño sobrio de una vivienda de un solo volumen, techumbre a dos aguas, colores exteriores representativos de la arquitectura mexicana y neutros en el interior. Cuenta con dos recámaras, un baño, un porche y la sala, comedor y cocina se concentran en un mismo espacio sin ningún elemento estructural que los divida. La orientación que se contempla para este estudio es con fachada principal al suroeste.

Figura 3. Vivienda emergente FONDEN en Guerrero, México
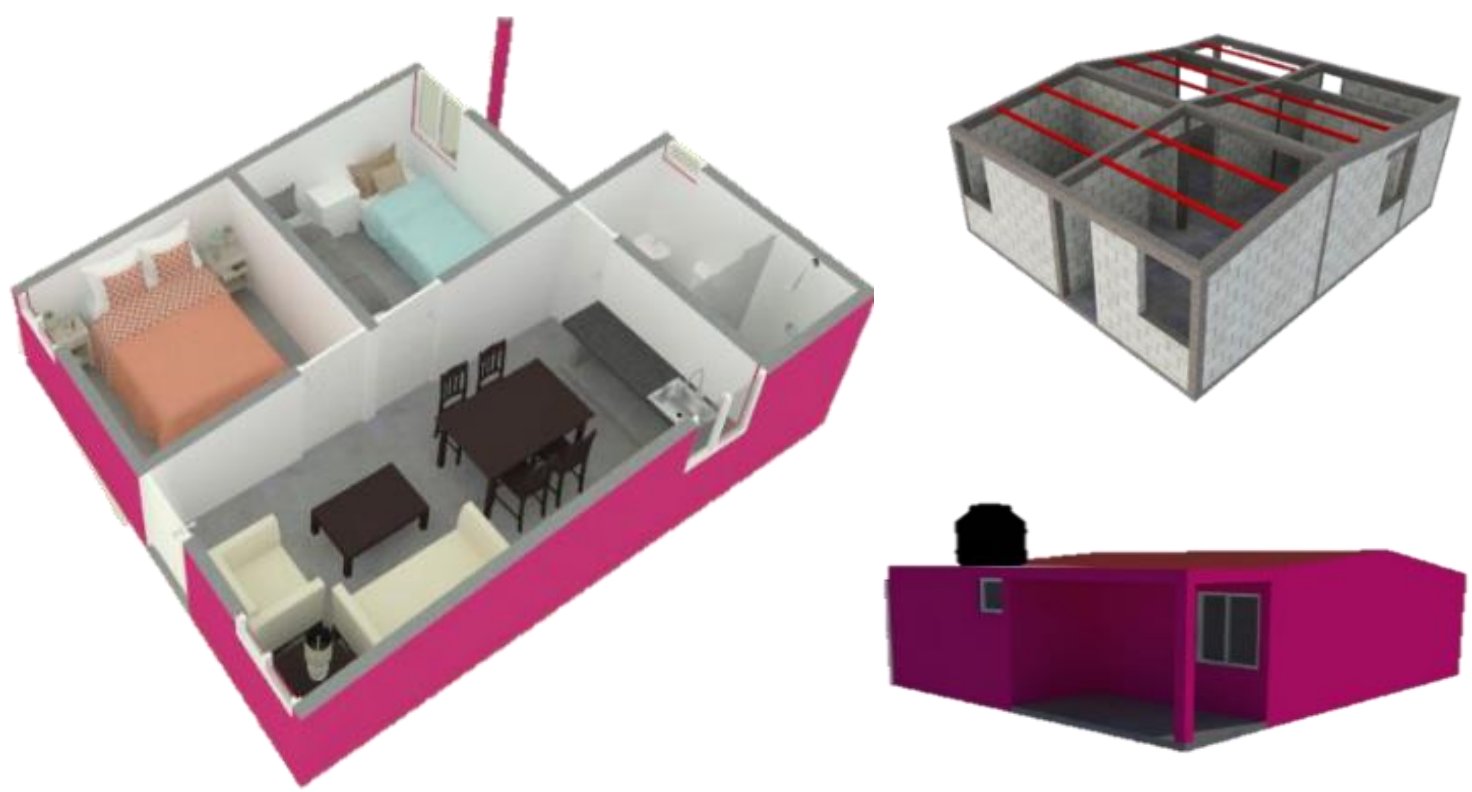

Fuente: Elaboración propia con referencia de las viviendas construidas en el Estado de Guerrero.

ACE, 16 (47) CC BY-ND 3.0 ES | UPC Barcelona, España | Propuesta para vivienda emergente con perspectiva ZIH en 
La vivienda (Figura 3) es entregada con puertas de multipanel, ventanas de vidrio simple y aluminio, y muebles fijos (WC, lavamanos, regadera y tarja en cocina). Se contempla con la instalación de todos los servicios (Luz, agua y drenaje) y un tinaco para su correcto funcionamiento.

Los materiales que se utilizan en el caso base son los estipulados en los lineamientos de FONDEN (Diario oficial de la federación, 2011): fundación de losa de cimentación $10 \mathrm{~cm}\left(\mathrm{U}=2.93 \mathrm{~W} / \mathrm{m}^{2} \mathrm{~K}\right)$, concreto armado (acabado cemento pulido), muros de block con revestimiento cemento arena $(U=$ $\left.1.30 \mathrm{~W} / \mathrm{m}^{2} \mathrm{~K}\right)$, techumbre de láminas tipo " $\mathrm{S}$ " de fibrocemento $\left(\mathrm{U}=5.94 \mathrm{~W} / \mathrm{m}^{2} \mathrm{~K}\right)$, para el baño es losa de concreto armado de 10 a $12 \mathrm{~cm}\left(U=2.73 \mathrm{~W} / \mathrm{m}^{2} \mathrm{~K}\right)$.

Para la hermeticidad en el software DesignBuilder se considera un valor n50 de 5,0 renovaciones de aire por hora (ach).

\section{Análisis de la vivienda FONDEN con metodología ZIH}

\subsection{Habitabilidad térmica (HT)}

Se analiza la vivienda desde dos perspectivas diferentes, la primera es con un rango de confort adaptativo y la segunda con un rango de confort de la NAMA, que es con la que evalúan las viviendas mexicanas. Como primera evaluación se analiza con un modelo de confort adaptativo (Szokolay, 2004). El modelo define la temperatura neutral (Tn) en base a la temperatura media (Tm) del mes a analizar.

Donde

$$
\operatorname{Tn}=17,6+0,31 \times \mathrm{Tm}
$$

Tn: Temperatura neutral $\left({ }^{\circ} \mathrm{C}\right)$.

Tm: Temperatura media del mes $\left({ }^{\circ} \mathrm{C}\right)$.

Este modelo plantea una amplitud de +/- 2.5, es decir;

Tinf: $\mathrm{Tn}-2,5^{\circ} \mathrm{C}$

Tsup: $\operatorname{Tn}+2,5^{\circ} \mathrm{C}$

Tinf: límite inferior de la temperatura operativa $\left({ }^{\circ} \mathrm{C}\right)$.

Tsup: límite superior de la temperatura operativa $\left({ }^{\circ} \mathrm{C}\right)$.

Considerando el análisis generado con el software Climate Consultant, se obtuvo la temperatura de bulbo promedio mensual, con ella y las fórmulas antes mencionadas, se obtuvo un rango de confort adaptativo mensual. Como segunda evaluación con la NAMA (SEMARNAT/CONAVI, 2012), plantea un rango de confort para todas las zonas climáticas del territorio nacional, este oscila en una temperatura que va de $\operatorname{los} 20^{\circ} \mathrm{C}$ a los $25^{\circ} \mathrm{C}$ y se puede llegar a extender hasta los $27.5^{\circ} \mathrm{C}$, pero las evaluaciones energéticas llegan a $25^{\circ} \mathrm{C}$.

\section{- HT- Confort adaptativo}

Al comparar todos los espacios de la vivienda emergente, las áreas comunes se consideran el espacio más perjudicado. Las habitaciones mostraron una leve diferencia una de otra, la más favorable fue la habitación 2 que tiene el porche por un costado y le ayuda en el control solar. El espacio más beneficioso de toda la vivienda es el baño que dobla en porcentaje - hora a los demás recintos.

Haciendo una comparativa entre las localidades, Ixtapilla mantiene un nivel de habitabilidad térmica más elevado que San Vicente, en ambos casos conservan el mismo orden de los recintos, más a menos privilegiados (Tabla 2), pero su porcentaje varía. 


\section{- HT - Confort NAMA}

Las localidades de San Vicente y Chilpancingo mantienen similitudes, teniendo al baño como su espacio más favorable y si se observa el rango de confort extendido en Chilpancingo, puede llegar a alcanzar HT de 90\%. En Ixtapilla es lo contrario, el baño presenta la HT más baja en comparativa con las demás, y en todos los recintos en verano el porcentaje de HT otorgado por el rango de confort extendido puede llegar a ser más significativo que el estándar (

Tabla 2).

En términos generales, el rango de confort extendido va incrementándose conforme aumenta la temperatura de la localidad, es decir, en el clima semifrío que representa San Vicente es menor el porcentaje de HT, se incrementa el porcentaje de HT en Chilpancingo, y en Ixtapilla.

El baño es el único recinto que tiene un cambio de material en su techumbre, y presenta diferencias elevadas. El material que se implementó en el baño, da más estabilidad en la frecuencia de temperatura operativa, que se mantiene en comparativa de los otros espacios, exceptuando el clima cálido evaluado por la NAMA. Se concluye que se tiene que cambiar como material la techumbre de fibrocemento que cubre gran porcentaje de la vivienda, para lograr estabilizar la frecuencia de la temperatura operativa.

\subsection{Energía}

En el Estado de Guerrero, las tres localidades analizadas cuentan con una baja probabilidad de presencia de equipos de aire acondicionado (Comisión Nacional para el Uso Eficiente de la Energía, 2016a), sin embargo, fue necesario analizar las viviendas con equipos de climatización debido a sus bajos índices de habitabilidad térmica.

Según los escenarios de la vivienda emergente FONDEN, considerando las condiciones de borde y rangos de confort $\left(20\right.$ a $25^{\circ} \mathrm{C}$ y set back de 18 a $28^{\circ} \mathrm{C}$ ) establecidos por la NAMA, se realizaron simulaciones en los diferentes climas con el software Design Builder. Para el factor de emisión del Sistema Eléctrico Nacional se considera 0.582 T de $\mathrm{CO}_{2} / \mathrm{MWh}$ (Comisión Reguladora de Energía, 2017).

Conforme a lo estipulado por la Comisión Federal de Electricidad (CFE, 2018) y la Comisión Nacional para el Uso Eficiente de la Energía 2016b se contempla que para las localidades de San Vicente y Chilpancingo de Los Bravo aplica la Tarifa 1, y en Ixtapilla, la tarifa 1E. También existe el servicio doméstico de alto consumo (DAC), generado cuando los kilowatts hora consumidos dentro del hogar son más de los programados para esa región, contemplado las variables de costo por temporadas de invierno y verano. En las tres localidades, hay que considerar que, para efectos de simulación, solo fueron considerados los requerimientos de refrigeración y calefacción, sin contemplar energía primaria de la vivienda (electrodomésticos, iluminación, equipos de hogar), los cuales elevarían aún más el consumo energético.

Las emisiones de $\mathrm{CO}_{2}$ alcanzadas por San Vicente y Chilpancingo, son de 3,470 y 3,271 $\mathrm{kg}$ de $\mathrm{CO}_{2}$ eq/año respectivamente, las cuales son menor a las emitidas por Ixtapilla, las cuales alcanzaron un valor de $5,967 \mathrm{~kg}$ de $\mathrm{CO}_{2}$ eq/año, además los costos por concepto de consumo de energía son superiores a los de Ixtapilla (Tabla 2). A saber, San Vicente alcanza un costo anual de \$30,622.99 MXN, es decir, el equivalente a 347 salarios mínimos diarios (el salario mínimo general diario en México 2018 es de \$88.36 MXN según el Diario Oficial de la Federación, 2018), de acuerdo a estos datos los usuarios tendrían que trabajar casi todo un año para cubrir solo el costo energético de climatización de su hogar.

ACE, 16 (4.7) CC BY-ND 3.0 ES | UPC Barcelona, España | Propuesta para vivienda emergente con perspectiva ZIH en 
Este tipo de sistema tarifario resulta favorable para climas como Ixtapilla, pero desfavorables para San Vicente y Chilpancingo, debido a que se consideran condiciones climáticas de altas temperaturas, más no bajas, y por consiguiente afecta a los usuarios al momento de considerar un equipo de calefacción. Solo el $2.6 \%$ de las viviendas mexicanas consideran equipos de esta índole (Comisión Nacional para el Uso Eficiente de la Energía, 2016b), esto se suma al hecho que Guerrero sea de los principales Estados con mayor rezago social, donde para el usuario pagar tal cantidad en climatización resulta imposible.

\subsection{Agua}

La vivienda está situada fuera de la mancha urbana capitalina. Examinados los usos y tradiciones que se viven en la zona, se calculan las emisiones de $\mathrm{CO}_{2}$ asociadas al consumo del agua. En el baño y cocina se contemplaron artefactos tradicionales, sin ahorro en agua y sin consumo energético; en la cocina se contempla hervir el agua debido a que no toda el agua de la red nacional es para consumo humano. Además, se considera un lavadero, suponiendo que primeramente se les darán prioridad a otros muebles de la vivienda. En el área de riesgo se consideró una cantidad acorde a las costumbres locales, debido a que en los lugares ejidales las personas tienden a generar un espacio para jardín, donde puedan tener algún tipo de planta; no se usa el césped, pero se acostumbra a rociar con agua la tierra por la mañana.

Con los datos agregados al SAAVI combinados al código postal de la localidad, Nuevo Azinyahualco, arroja un consumo proyectado de agua de 420.40 m3/vivienda*año, el equivalente a un 13.6\% sobre la línea base. Respecto a las emisiones de $\mathrm{CO}_{2}$ estas ascienden a $349.46 \mathrm{~kg} \mathrm{CO}$ eq/vivienda*año, lo que está un $0.6 \%$ sobre la línea base1. El costo para el usuario por mantener este tipo de vivienda por $\mathrm{m}^{3}$ de agua para la región de Chilpancingo de Los Bravo, Guerrero, es de \$11.38 MXN (CONAGUA, 2018) lo que significaría $\$ 4,784.15 \mathrm{MXN}$ anuales, equivalente a 54 veces el salario mínimo diario, casi dos meses para cubrir solo el consumo de agua.

\subsection{Uso de suelo}

A las personas damnificadas de la localidad de Azinyahualco se les otorgó una vivienda emergente FONDEN a 10 kilómetros de donde ellos residían (Figura 4), cercanos a la localidad de Zoyatepec y, aun así, se les llamo "Nuevo Azinyahualco".

El lugar donde residían en un inicio las personas damnificadas era catalogado de alto riesgo, esta fue la razón para generar una reubicación geográfica. En esta investigación consideramos que el lugar debió ser evaluado, teniendo en cuenta un emplazamiento más cercano a su localidad, ya que a las víctimas se les relaciona con la idea de permanecer en su comunidad después del desastre (G. Zhang, Setunge, \& van Elmpt, 2014) y sentir que algo aún les pertenece (Cruz Roja - Media Luna Roja, 2011). Bajo estos argumentos se cree que los damnificados debieron sentir una desventaja, no solo por perder todas sus pertenecías, sino también por alejarse del lugar que alguna vez albergo su hogar.

Para la construcción que se realizó se hizo uso de un terreno de 30,000 $\mathrm{m}^{2}$, para edificar las 93 viviendas requeridas y se removió total e ilegalmente la vegetación forestal, por lo que la Procuraduría Federal de Protección al Ambiente (PROFEPA, 2015) los clausuró de manera temporal en el año 2015, el motivo fue no cumplir con el cambio de uso de suelo, que es emitida por la Secretaría de Medio Ambiente y Recursos Naturales (SEMARNAT).

\footnotetext{
${ }^{1}$ La línea base utilizada en la herramienta SAAVi era la vivienda tipo construida en el 2010, y es la base que se utiliza para comparar el progreso de las nuevas construcciones de la vivienda mexicana.
} 


\section{ACE Architecture, City and Environment}

Figura 4. Vista aérea de la ubicación de las localidades Zoyatepec y Azinyahualco

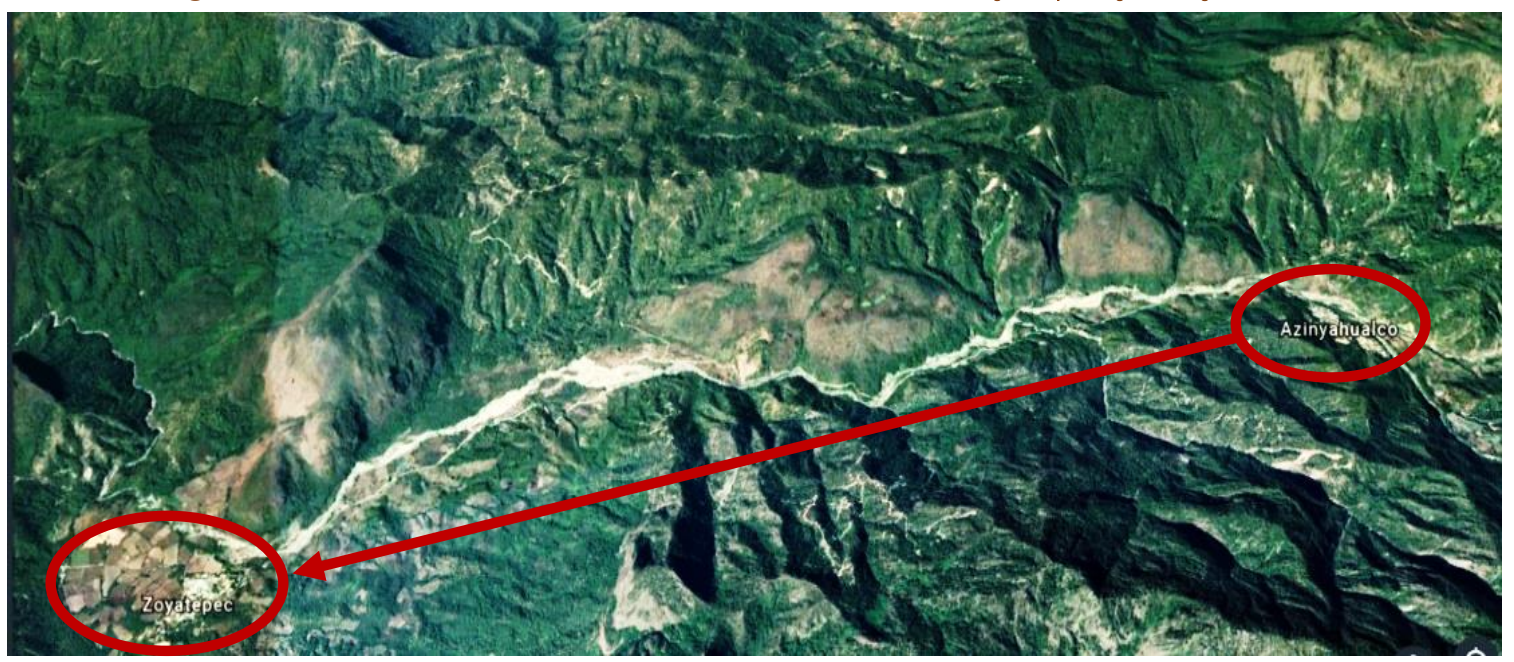

Fuente: Google maps 2018.

Existen emisiones asociadas al cambio de uso de suelo del Nuevo Azinyahualco, porque antes de albergar la construcción, el terreno contenía un bosque de pino-encino, lo que le otorgaba una calidad forestal por la capacidad de absorción de $\mathrm{CO}_{2}$ y al edificarse la parcela quedo biológicamente improductiva.

Para el cálculo de la huella ecológica (Martínez Rocamora et al., 2017) por la superficie consumida se considera la siguiente expresión:

$$
\text { HESUP }=S \times F E b
$$

Donde:

HEsup = Huella de superficie consumida (hag)

$\mathrm{S}=$ Superficie consumida (ha)

$\mathrm{FEb}=$ Factor de equivalencia de los bosques (1.29 hag / há) (Global Footprint Network National Footprint Accounts, 2018)

Por lo que:

HEsup $=3$ ha $\times 1.29$ hag $/$ ha

HEsup $=3.87$ hag

La huella del Nuevo Azinyahualco es de 3,87 hag, pero al hacer uso de la parcela sin existir un cambio de uso de suelo aprobado con anterioridad, se genera otro tipo de desastre, por eso, es primordial contemplar una estrategia de acción antes de un desastre que incluya una mejor planeación para casos de reubicación.

Tabla 2. Análisis ZIH caso base

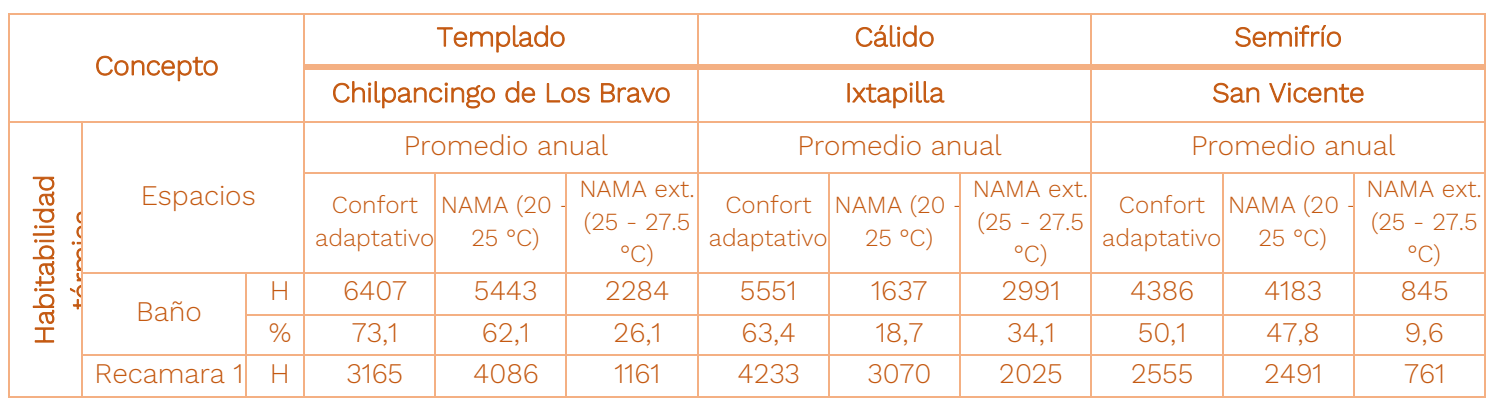

ACE, 16 (47) CC BY-ND 3.0 ES | UPC Barcelona, España | Propuesta para vivienda emergente con perspectiva ZIH en 


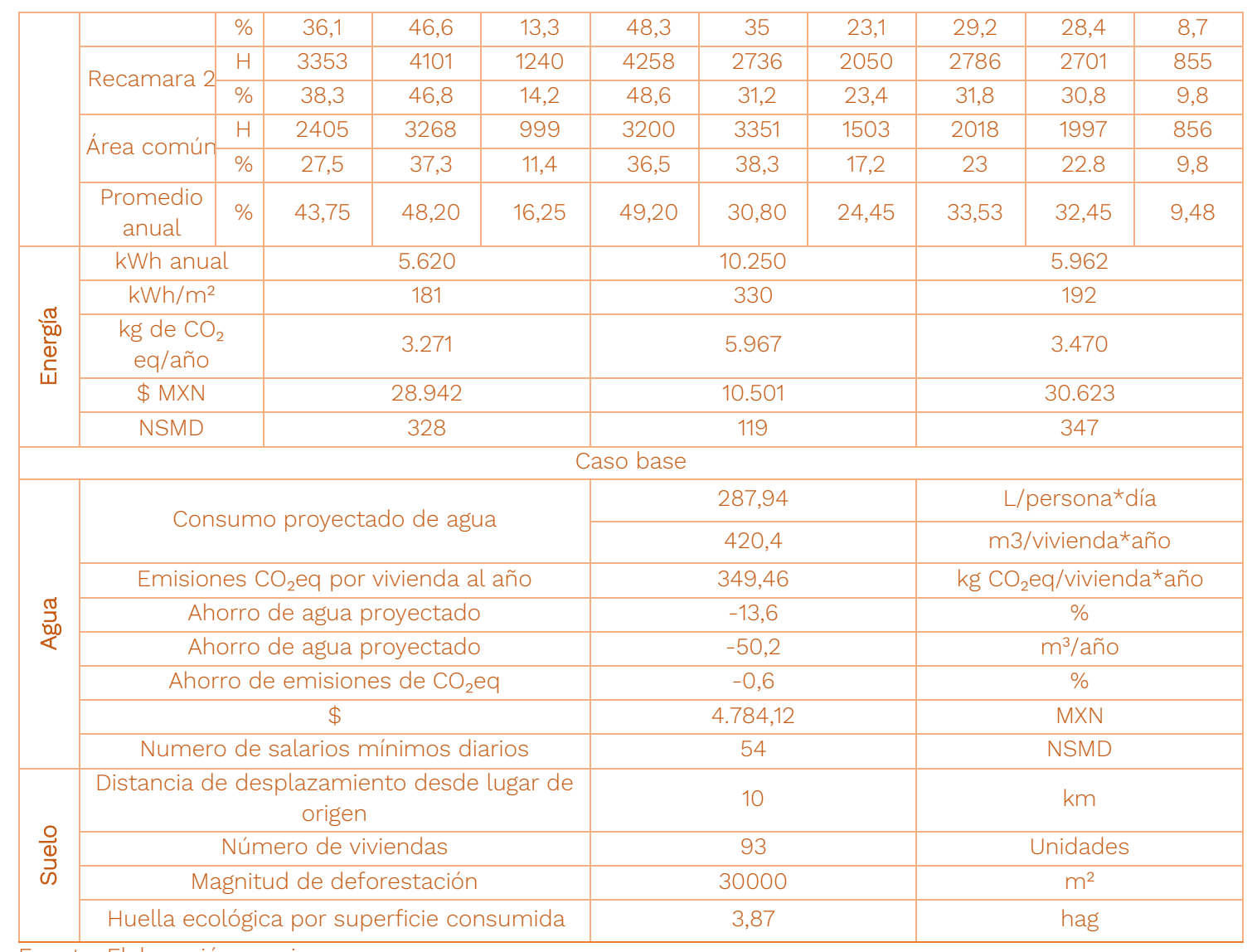

Fuente: Elaboración propia.

\section{Comparativa con la NAMA}

Se desarrolla una comparación con los resultados obtenidos en el caso base de la vivienda emergente FONDEN y los desarrollados por la NAMA de vivienda sustentable en México. Se utilizan tres modelos; el primer modelo pertenece a un "caso base" realizado por Campos (Campos Arriaga, 2011) a nombre de y apoyado por GIZ/GOPA, donde se utilizaron sistemas constructivos convencionales, al igual que los diseños de construcción de vivienda social más populares del mercado actual mexicano (SEMARNAT/CONAVI, 2012).

Para la elaboración de la NAMA, que se toma como referencia en esta investigación, participo Passive House Institute, que utiliza el mismo modelo de construcción habitual (Caso base), con diferentes parámetros constructivos, representando así el segundo modelo de esta comparativa. Los climas que representan el caso base y Passive House se consideran similares a los lugares estudiados en la vivienda emergente.

\subsection{Habitabilidad térmica}

Al analizar ambos casos, San Vicente cuenta con menor número de horas - confort - año que Puebla, donde su espacio más desfavorable es su área común o pública al contar con $22.80 \%$ de horas confort - año, mientras que el caso base se encuentra en $45.34 \%$. 
En el clima cálido las viviendas tuvieron un comportamiento relacionado con la demanda energética del punto anterior. Ixtapilla maneja rangos de HT entre 31.20 a 38.30\% mientras que Cancún sus rangos se encuentran entre 27.80 a 33.36\%, encontrándose un poco más desfavorable que Ixtapilla. La comparativa de los clima cálido y semifrío se hizo con el caso base de una vivienda tradicional del 2011, no con los estándares Passive House o ecodiseños, que se proponen en la NAMA.

\subsection{Energía}

En la Tabla 3 muestra que la vivienda emergente FONDEN es muy similar al caso base de vivienda popular que se construía en el 2010 y totalmente contrapuesta a los planes y perspectivas a futuro que tiene CONAVI para la vivienda mexicana con la Passive House. Suponiendo que el abastecimiento energético será a partir de la red eléctrica, con el consecuente aumento en las emisiones de $\mathrm{CO}_{2}$, que de alcanzar $5.82 \mathrm{~kg} \mathrm{CO}_{2}$ eq/año* $\mathrm{m}^{2}$ en clima templado, 9.31 en semifrío y pasa a 22.70 en clima cálido, la vivienda emergente se mantiene por sobre los $100 \mathrm{~kg} \mathrm{CO}_{2}$ eq/año*m², con 105.37 (templado), 111.78 (semifrío) y 192.23 (cálido) kg CO 2 eq/año*m².

\subsection{Agua}

Para la línea base se manejan artefactos tradicionales sin ningún grado ecológico, y a partir de estos se empiezan a generar comparaciones con las dos tipologías de vivienda. En la vivienda NAMA, se consideran artefactos con grado ecológico; el baño, un WC de 5 L/descarga, un grifo de 6 L/min y regadera de $3.8 \mathrm{~L} / \mathrm{min}$. En la cocina se utiliza un grifo de $6 \mathrm{~L} / \mathrm{min}$. Al hacer la comparación en la Tabla 3, se muestra que la vivienda emergente se encuentra debajo de la línea base, y en el $\mathrm{CO}_{2}$ eq la vivienda emergente FONDEN tiene un $0.6 \%$ sobre la línea base, mientras que la NAMA tienen un ahorro de $0.9 \%$

Tabla 3. Comparativa con la NAMA

\begin{tabular}{|c|c|c|c|c|c|c|c|c|c|c|c|}
\hline \multirow{2}{*}{\multicolumn{3}{|c|}{ Concepto }} & \multicolumn{3}{|c|}{ Templado } & \multicolumn{3}{|c|}{ Cálido } & \multicolumn{3}{|c|}{ Semifrío } \\
\hline & & & \multicolumn{3}{|c|}{ Chilpancingo de Los Bravo } & \multicolumn{3}{|c|}{$\begin{array}{l}\text { Promedio anual NAMA (20 } \\
\left.-25^{\circ} \mathrm{C}\right)\end{array}$} & \multicolumn{3}{|c|}{$\begin{array}{c}\text { Promedio anual NAMA (20 } \\
\left.\qquad-25^{\circ} \mathrm{C}\right)\end{array}$} \\
\hline \multirow{8}{*}{ 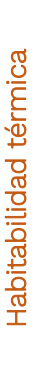 } & \multicolumn{2}{|c|}{ Espacios } & & & & \multicolumn{2}{|c|}{ Ixtapilla } & Cancún & \multicolumn{2}{|c|}{ San Vicente } & Puebla \\
\hline & \multirow{2}{*}{ Área común } & $\mathrm{H}$ & & & & \multicolumn{2}{|c|}{3351} & 2922 & \multicolumn{2}{|c|}{1997} & 3972 \\
\hline & & $\%$ & & & & \multicolumn{2}{|c|}{38,3} & 33,36 & \multicolumn{2}{|c|}{22,8} & 45,34 \\
\hline & \multirow{2}{*}{ Recamara 1} & $\mathrm{H}$ & & & & \multicolumn{2}{|c|}{3070} & 2435 & \multicolumn{2}{|c|}{2491} & 4101 \\
\hline & & $\%$ & & & & \multicolumn{2}{|l|}{35} & 27,8 & \multicolumn{2}{|c|}{28,4} & 46,82 \\
\hline & \multirow{2}{*}{ Recamara 2} & $\mathrm{H}$ & & & & \multicolumn{2}{|c|}{2736} & 2755 & \multicolumn{2}{|c|}{2701} & 3860 \\
\hline & & $\%$ & & & & \multicolumn{2}{|c|}{31,2} & 31,45 & \multicolumn{2}{|c|}{30,8} & 44,06 \\
\hline & $\begin{array}{l}\text { Promedio } \\
\text { anual }\end{array}$ & $\%$ & & & & \multicolumn{2}{|c|}{26,13} & 23,15 & \multicolumn{2}{|c|}{20,50} & 34,06 \\
\hline \multirow{7}{*}{ 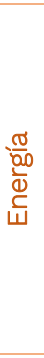 } & \multicolumn{2}{|c|}{ Vivienda Tipo } & $\begin{array}{l}\text { Caso } \\
\text { base }\end{array}$ & $\begin{array}{l}\text { Passive } \\
\text { house }\end{array}$ & $\begin{array}{l}\text { Viv. } \\
\text { FONDEN }\end{array}$ & $\begin{array}{l}\text { Caso } \\
\text { base }\end{array}$ & $\begin{array}{l}\text { Passive } \\
\text { house }\end{array}$ & $\begin{array}{l}\text { Viv. } \\
\text { FONDEN }\end{array}$ & $\begin{array}{l}\text { Caso } \\
\text { base }\end{array}$ & $\begin{array}{l}\text { Pass } \\
\text { hol }\end{array}$ & $\begin{array}{l}\text { Viv. } \\
\text { FONDEN }\end{array}$ \\
\hline & \multirow{2}{*}{\multicolumn{2}{|c|}{$\begin{array}{l}\text { Dem. Calef. } \\
\text { kWh/ m² año }\end{array}$}} & \multirow[t]{2}{*}{38} & \multirow[t]{2}{*}{4} & \multirow[t]{2}{*}{22,17} & 0 & 0 & 0,07 & 187 & 15 & 121,6 \\
\hline & & & & & & & & & & & \\
\hline & Dem. Refr & & 124 & 6 & 158,88 & 506 & 39 & 330,22 & 72 & 1 & 70,46 \\
\hline & $\mathrm{kWh} / \mathrm{m}^{2} \mathrm{a}$ & & 124 & 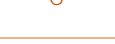 & ט, & 50 & & & & & \\
\hline & Emisione & & & & & & & & & & \\
\hline & $\begin{array}{r}\mathrm{kg} \mathrm{CO} \mathrm{CO}_{2} \\
\text { eq/año*m }\end{array}$ & & 94,28 & 5,82 & 105,37 & 294,49 & 22,7 & 192,23 & 150,74 & 9, & 111,78 \\
\hline & & & & & & so base & & & & & \\
\hline
\end{tabular}

ACE, 16 (47) CC BY-ND 3.0 ES | UPC Barcelona, España | Propuesta para vivienda emergente con perspectiva ZIH en 


\begin{tabular}{|c|c|c|c|c|c|}
\hline \multirow{7}{*}{ 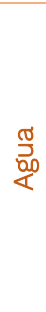 } & Concepto & $\begin{array}{l}\text { Línea } \\
\text { base }\end{array}$ & NAMA & $\begin{array}{c}\text { Viv. } \\
\text { FONDEN }\end{array}$ & Unidades \\
\hline & \multirow{2}{*}{ Consumo proyectado de agua } & 253,6 & 198,8 & 287,94 & L/persona*día \\
\hline & & 370,2 & 290,24 & 420,4 & m3/vivienda*año \\
\hline & Emisiones $\mathrm{CO}_{2}$ eq por vivienda al año & 347,54 & 344,46 & 349,46 & $\mathrm{~kg} \mathrm{CO}{ }_{2}$ eq/vivienda*año \\
\hline & Ahorro de agua proyectado & $x$ & 21,6 & $-13,6$ & $\%$ \\
\hline & Ahorro de agua proyectado & $x$ & 80 & $-50,2$ & $\mathrm{~m}^{3} / \mathrm{año}$ \\
\hline & Ahorro de emisiones de $\mathrm{CO}_{2} \mathrm{eq}$ & $x$ & 0,9 & $-0,6$ & $\%$ \\
\hline
\end{tabular}

Fuente: Elaboración propia.

\subsection{Factibilidad}

Según las comparaciones que se realizaron, se constata que la vivienda emergente FONDEN se encuentra debajo de las proyecciones que se están considerando en la vivienda por parte de CONAVI, incluso, en algunos casos se encuentran por debajo de la línea base. Con ello, se genera una preocupación al constatar que se dañaron 51,137 viviendas en solo siete años en el Estado de Guerrero (Tabla 13) y se restauran o reconstruyen fuera de las proyecciones de la NAMA.

Lo anterior genera una incertidumbre en los escenarios de la NAMA que tienen proyectados para la vivienda social en México, más aún cuando las viviendas de emergencias FONDEN nacen de una catástrofe, pero están construidas para ser definitivas.

\section{Recomendaciones para el caso base}

\subsection{Materiales}

Muros: Se proponen bloques de tierra compactada (BTC), usando para la unión un mortero de cal hidráulica natural. Estos bloques cuentan con resistencia a la compresión de $40 \mathrm{Kg} / \mathrm{cm}^{2}$, que es muy similar al ladrillo rojo recocido (FLORES, 2015) y conductividad térmica de $0.778 \mathrm{~W} / \mathrm{m} \cdot \mathrm{K}$ (SAINZ \& DE TORRES, n.d.).

Cubierta: Se propone concreto reciclado con $f^{\prime} \mathrm{c}=250 \mathrm{~kg} / \mathrm{cm}^{2}$, elaborado con agregado grueso de un $25 \%$ de reciclado - $75 \%$ árido natural, y agregado fino 25\% reciclado - $75 \%$ árido natural, permaneciendo prácticamente igual su desempeño de resistencia, porosidad y costo (Bedoya \& Dzul, 2015). Que según estudios de laboratorio es un $K=1.425 \mathrm{~W} / \mathrm{m} \mathrm{K}$.

Ventanas: Se propone una película para control solar en las ventanas, para reducir las ganancias solares.

\subsection{Habitabilidad térmica}

Se concluye que la propuesta de vivienda de emergencia, en los tres climas evaluados, con el rango de confort estándar de la NAMA y el adaptativo, al implementarla, se tendría un mejor comportamiento en un clima templado, ya que se tiene $60 \%$ de HT anual en el baño y los demás espacios están por encima del $80 \%$ de HT anual. Debemos subrayar que, el único clima que tuvo mayor aceptación en todos sus recintos al ser mayor de 80\% la HT anual, es en clima cálido de Ixtapilla (Tabla 4). 
Aunque en San Vicente la recámara 1 y los espacios comunes muestran la HT anual más baja, en diciembre, los espacios comunes mantienen la HT en un rango de 15.59\%, siendo la más baja y en abril, 68.89\% como la más alta; mientras la recámara 1 tiene 0\% en diciembre - enero como lo más bajo y $74.33 \%$ en mayo como lo más alto.

La frecuencia operativa del caso base en la recámara 1, (como se puede observar en la Figura 5 y Figura 6 del mes de enero), tiene mínimos de $11^{\circ} \mathrm{C}$ y podía llegar a $28^{\circ} \mathrm{C}$; mientras en la propuesta se mantiene entre mínimas de $15^{\circ} \mathrm{C}$ y máximas de $20^{\circ} \mathrm{C}$. Concluimos que, aunque en la propuesta la $\mathrm{HT}$ es nula o baja, existe un mejor comportamiento térmico al tener una frecuencia de temperatura operativa más delimitado.

Figura 5. Frecuencia de temperatura operativa del mes de enero de la Recámara 1 (San Vicente), en vivienda de emergencia caso base

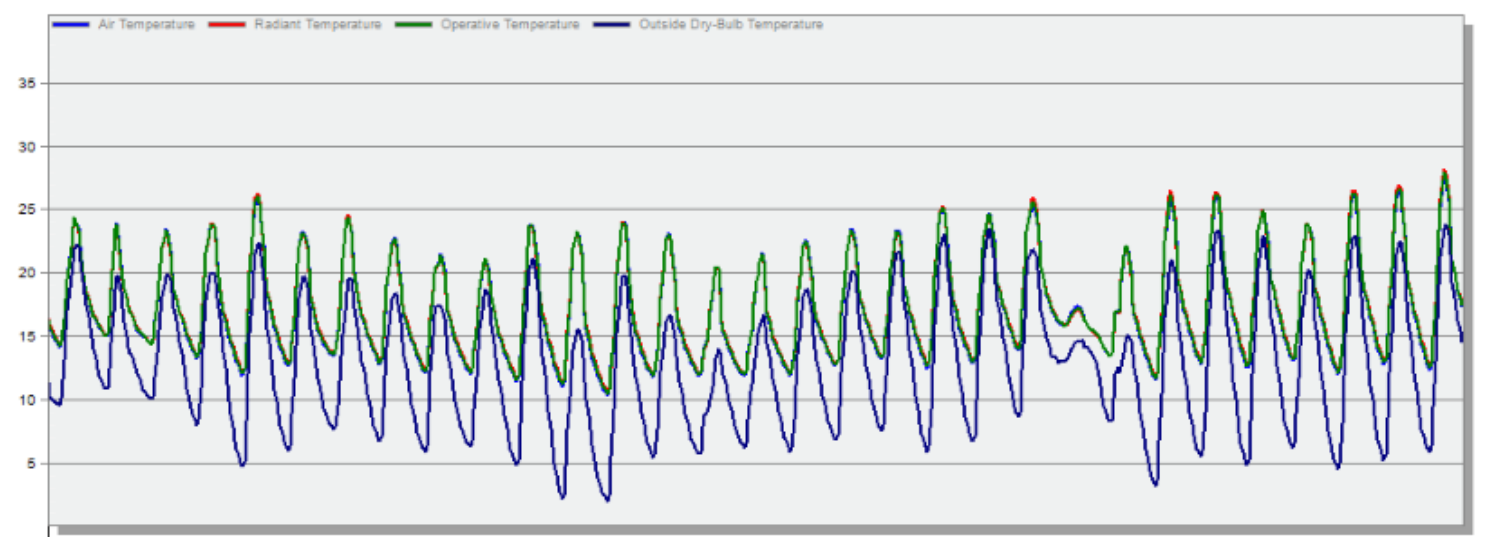

Fuente: Elaboración propia utilizando el software Design Builder.

Figura 6. Frecuencia de temperatura operativa del mes de enero de la Recámara 1 (San Vicente), en vivienda de emergencia propuesta.

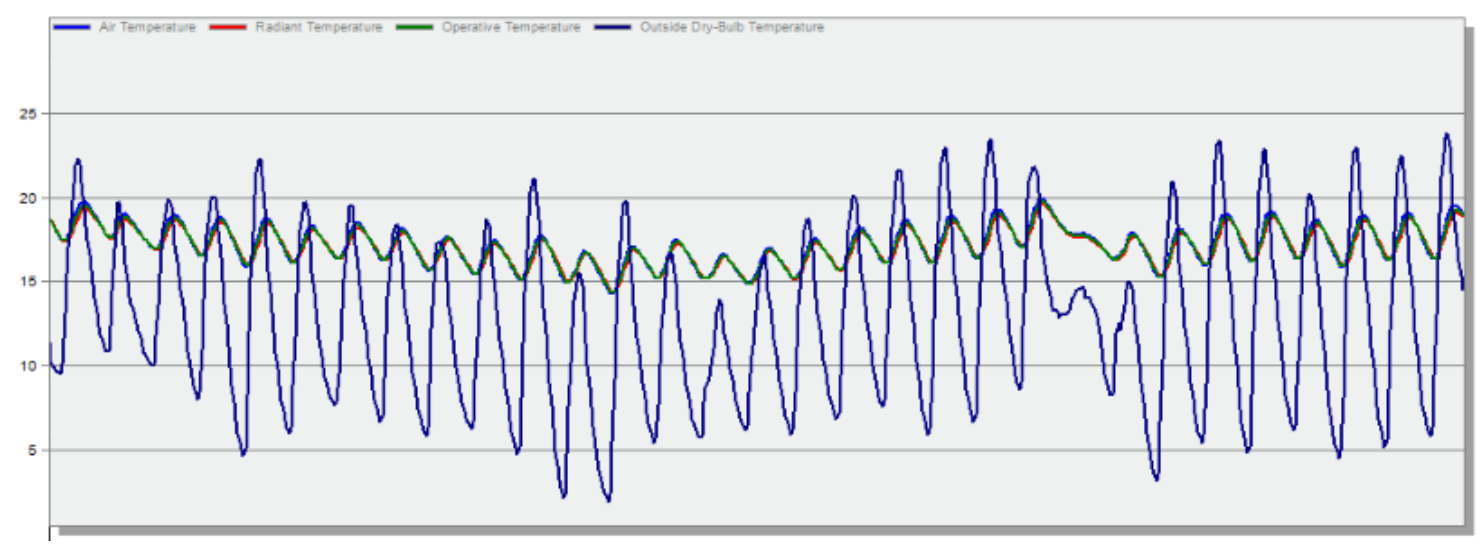

Fuente: Elaboración propia utilizando el software Design Builder.

A pesar que se realizaron evaluaciones de habitabilidad térmica con rangos de confort estipulados por la NAMA (estándar y extendido), se considera en esta investigación que, lo más viable para esta región de Guerrero, es el análisis con un rango de confort adaptativo, debido a que las personas locatarias tienen diferentes condiciones climáticas, y en algunas zonas las costumbres sociales son muy arraigadas, por lo que no se debería generalizar a todos en una misma neutralidad térmica cuando los usuarios tienen diferentes condicionantes. 


\subsection{Energía}

Estos resultados exhiben una disminución respecto al caso base de la vivienda de emergencia de FONDEN en Guerrero, donde presentaban un costo por el consumo energético en Chilpancingo de 328 NSMD, Ixtapilla 119 NSMD y San Vicente con 347 NSMD y se redujeron a 2, 8 y 13 NSMD. Las emisiones proyectadas que generaría esta propuesta se comparan a las perspectivas que tiene la NAMA, especialmente al tipo "Passive House" (Tabla 4).

Tabla 4. Análisis ZIH de las recomendaciones de la vivienda emergente

\begin{tabular}{|c|c|c|c|c|c|c|c|c|c|c|c|}
\hline \multirow{2}{*}{\multicolumn{3}{|c|}{ Concepto }} & \multicolumn{3}{|c|}{ Templado } & \multicolumn{3}{|c|}{ Cálido } & \multicolumn{3}{|c|}{ Semifrío } \\
\hline & & & \multicolumn{3}{|c|}{ Chilpancingo de Los Bravo } & \multicolumn{3}{|c|}{ Ixtapilla } & \multicolumn{3}{|c|}{ San Vicente } \\
\hline \multirow{11}{*}{ 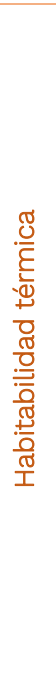 } & \multirow{2}{*}{\multicolumn{2}{|c|}{ Espacios }} & \multicolumn{3}{|c|}{ Promedio anual } & \multicolumn{3}{|c|}{ Promedio anual } & \multicolumn{3}{|c|}{ Promedio anual } \\
\hline & & & $\begin{array}{c}\text { Confort } \\
\text { adaptativo }\end{array}$ & $\begin{array}{c}\text { NAMA }(20 \\
\left.25^{\circ} \mathrm{C}\right)\end{array}$ & $\begin{array}{c}\text { NAMA ext. } \\
(25-27.5 \\
\left.{ }^{\circ} \mathrm{C}\right)\end{array}$ & $\begin{array}{c}\text { Confort } \\
\text { adaptativo }\end{array}$ & $\begin{array}{c}\text { NAMA }(20 \\
\left.25^{\circ} \mathrm{C}\right)\end{array}$ & $\begin{array}{c}\text { NAMA ext. } \\
(25-27.5 \\
\left.{ }^{\circ} \mathrm{C}\right)\end{array}$ & $\begin{array}{c}\text { Confort } \\
\text { adaptativo }\end{array}$ & $\begin{array}{c}\text { NAMA }(20 \\
\left.25^{\circ} \mathrm{C}\right)\end{array}$ & $\begin{array}{c}\text { NAMA ext. } \\
(25-27.5 \\
\left.{ }^{\circ} \mathrm{C}\right)\end{array}$ \\
\hline & \multirow{2}{*}{ Baño } & $\mathrm{H}$ & 7553 & 5464 & 2806 & 7932 & 883 & 4209 & 4478 & 6097 & 70 \\
\hline & & $\%$ & 86,22 & 62,37 & 32,03 & 90,55 & 10,08 & 48,05 & 51,12 & 69,60 & 0,80 \\
\hline & \multirow{2}{*}{ Recamara 1} & $H$ & 5562 & 7655 & 698 & 7840 & 4359 & 3784 & 3530 & 3318 & 1 \\
\hline & & $\%$ & 63,49 & 87,39 & 7,97 & 89,50 & 49,76 & 43,20 & 40,30 & 37,88 & 0,01 \\
\hline & \multirow{2}{*}{ Recamara 2} & $\mathrm{H}$ & 7521 & 7720 & 1006 & 8375 & 3606 & 4707 & 6895 & 5921 & 0 \\
\hline & & $\%$ & 85,86 & 88,13 & 11,48 & 95,61 & 41,16 & 53,73 & 78,71 & 67,59 & 0,00 \\
\hline & \multirow{2}{*}{ Área común } & $\mathrm{H}$ & 6105 & 7869 & 740 & 7115 & 4187 & 4058 & 6486 & 3986 & 0 \\
\hline & & $\%$ & 69,69 & 89,83 & 8,45 & 81,22 & 47,80 & 46,32 & 74,04 & 45,50 & 0,00 \\
\hline & $\begin{array}{c}\text { Promedio } \\
\text { anual }\end{array}$ & $\%$ & 76,32 & 81,93 & 14,98 & 89,22 & 37,20 & 47,83 & 61,04 & 55,14 & 0,20 \\
\hline \multirow{6}{*}{$\frac{\stackrel{\sigma}{\frac{\sigma}{0}}}{\frac{1}{\Phi}}$} & \multicolumn{2}{|c|}{ kWh anual } & \multicolumn{3}{|c|}{214} & \multicolumn{3}{|c|}{$1.052,09$} & \multicolumn{3}{|c|}{$1.020,72$} \\
\hline & \multicolumn{2}{|l|}{$\mathrm{kWh} / \mathrm{m}^{2}$} & \multicolumn{3}{|c|}{6,88} & \multicolumn{3}{|c|}{33,89} & \multicolumn{3}{|c|}{32,88} \\
\hline & \multicolumn{2}{|c|}{$\begin{array}{c}\text { kg de } \mathrm{CO}_{2} \\
\text { eq/año }\end{array}$} & \multicolumn{3}{|c|}{124,33} & \multicolumn{3}{|c|}{612,32} & \multicolumn{3}{|c|}{594,59} \\
\hline & $\begin{array}{l}\mathrm{kg} \text { de } \mathrm{co} \\
\text { eq/año*m }\end{array}$ & & & 4,01 & & & 19,72 & & & 19,16 & \\
\hline & $\$ M X N$ & & & 169,41 & & & 19,72 & & & $1.138,44$ & \\
\hline & NSMD & & & 1,92 & & & 7,80 & & & 12,88 & \\
\hline & & & & & & aso base & & & & & \\
\hline & & & & & & & 137,73 & & & persona* & \\
\hline & (2) & 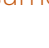 & proyecta & a de & & & 201,09 & & m3/ & /vivienda* & año \\
\hline & Emisior & es C & $\mathrm{O}_{2}$ eq por & vivienda a & año & & 12,58 & & $\mathrm{~kg} \mathrm{CO}$ & eq/vivien & a*año \\
\hline$\widetilde{\sigma}$ & Ah & rro & de agua p & royectadc & & & 45,70 & & & $\%$ & \\
\hline$\underset{x}{20}$ & Ah & rro & de agua p & royectadc & & & 169,1 & & & $\mathrm{~m}^{3} / a$ ño & \\
\hline & Ahor & $O d$ & e emisione & es de $\mathrm{CO}_{2}$ & & & 96,40 & & & $\%$ & \\
\hline & & & $\$$ & & & & $2.288,40$ & & & MXN & \\
\hline & Numer & de & salarios $\mathrm{m}$ & hínimos di & arios & & 26 & & & NSMD & \\
\hline
\end{tabular}

Fuente: Elaboración propia. 


\subsection{Agua}

Se realizaron simulaciones en el abastecimiento de agua en Zoyatepec donde, se cambiaron los artefactos de la vivienda emergente FONDEN por grado ecológico, así como la opción de hervir agua por ósmosis inversa y el aprovechamiento de aguas lluvia para riego y limpieza. Con lo que se obtienen resultados favorables, tanto el ahorro de agua proyectado, que es del $45.7 \%$ y del ahorro de emisiones de $\mathrm{CO}_{2} \mathrm{eq}$, que es de 96.4\% (Tabla 4). Los artefactos fueron los que influyeron en el porcentaje de ahorro del consumo de agua y el TAV ósmosis inversa fue el que influyó en el 94\% de ahorro de emisiones de $\mathrm{CO}_{2}$ eq.

\subsection{Suelo}

Aunque el uso de suelo fue analizado en el caso base, es necesario una investigación independiente, que integre las políticas urbanas existentes en territorio nacional, con la huella ecológica de las posibles áreas a ocupar para desplace de damnificados.

Se considera que un análisis más detallado en mapas de riesgo, unido a las políticas urbanas con una visión de desarrollo sustentable, podría generar que la vivienda emergente FONDEN tuviese un logro definitivo y no transitorio, solo visto desde un enfoque por emplazamiento.

\section{Conclusiones}

Los desastres naturales son cíclicos, repitiéndose año a año, lo que debe conllevar a una revisión y mejora a las acciones y estrategias adoptadas por parte del Estado, para atender este tipo de contingencias atendiendo todos los aspectos multifactoriales en este estudio: dimensión social, ambiental y económica.

La metodología ZIH utilizada en esta investigación, ha permitido identificar los sectores sociales más vulnerables y a partir de ello, crear recomendaciones precisas a puntos específicos, entre los cuales se tienen:

1. Se siguen manteniendo lineamientos por parte de FONDEN donde los materiales para las cubiertas sean de láminas tipo "S" a base de fibrocemento, metálicas galvanizado o de cartón embreado, en un lugar donde han existido de 262 a 295 ciclones en un lapso de 1949 a 2015 (CENAPRED, 2018a), esto explicaría una de las razones de por qué se llegaron a dañar 51,157 viviendas en el lapso de estudio. Es imprescindible cambiar los lineamientos FONDEN en cuanto a materialidad de la envolvente para cambiar este hecho.

2. Es necesario aplicar otro tipo de planeación, psicología en el diseño y construcción de esta. Las viviendas construidas por parte de FONDEN son del género de emergencia, existen intenciones de ser definitivas y en algunos casos ha sido posible, pero existen casos no exitosos con $0 \%$ de ocupación. Para obtener una aceptación de las viviendas por parte de los damnificados, es necesario restructurar los actuales lineamientos, poniendo mayor énfasis a una planeación previa a los acontecimientos, para mejorar la rapidez constructiva y un análisis cultural de la zona para que el diseño, materialidad y sistema construido implementado pueda acogerse a las costumbres locales.

3. Existe pobreza energética en la zona. Las viviendas tienen baja habitabilidad térmica en sus espacios, la solución no es entregar equipos de climatización (aire acondicionado) a zonas de 
estratos sociales bajos debido a que la demanda energética en climatización para las viviendas emergentes FONDEN es de $181.06 \mathrm{KWh} / \mathrm{m}^{2}$ en clima templado, $330.33 \mathrm{KWh} / \mathrm{m}^{2}$ en clima cálido y $192.06 \mathrm{KWh} / \mathrm{m}^{2}$ (energía distrital) en clima semifrío, ¿Cómo sería posible pagarlo para un padre de familia con el salario mínimo? Además ¿Cómo es posible pagarlo cuando con estos rangos se pasa al sistema tarifario DAC? La solución es cambiar las condiciones térmicas internas, para ello es necesario cambiar la materialidad estipulada en los lineamientos. En el estudio se muestra que con la modificación de solo tres elementos en la envolvente tiene una mejora significativa la vivienda.

4. Los resultados de este estudio, muestran la incertidumbre respecto a la duración de la vivienda de emergencia y la calidad de vida que otorga a los usuarios, aunque se reconocen los esfuerzos en el actuar de FONDEN es necesario una reestructuración. La vivienda no debería elevar el nivel de vulnerabilidad del inquilino ante un fenómeno meteorológico, ni soportar microclimas que pudieran afectar su calidad de vida.

5. Se tienen conflictos con las mismas dependencias, además se desconoce el avance en el sector vivienda y los compromisos internacionales. Es incongruente que FONDEN llegue a tener conflictos con otras dependencias como PROFEPA, o que habiendo tantos avances en materia de vivienda sustentable en México no se estén considerando en sus lineamientos. Más que una actualización independiente por parte de FONDEN es necesario la consideración de otras dependencias en la misma mesa como CONAVI, Sociedad Hipotecaria Federal.

Por lo tanto, aunque el estudio hace una evaluación detallada de la vivienda FONDEN en tres climas de México y se hace una sugerencia del cambio de tres materiales de la envolvente para obtener resultados favorables, hay que considerar que los climas estudiados no son los más extremos de México, que hay diversos fenómenos naturales que tienen su nivel de impacto de zona a zona y, que México se distingue en ser un país rico culturalmente. Con esto, hay que considerar que es inminente la necesidad de cambios en los lineamientos FONDEN, pero solo se ha estudiado una entidad federativa de 32, por lo que se sugiere tener lineamientos estatales y tener antecedentes para hacer lineamientos federales.

\section{Agradecimientos}

Los autores agradecen al Dr. Alexis Perez F, al Dr. Gabriel Cereceda B y a Roberto Arriagada B., M.Sc., por su apoyo en el desarrollo de las actividades de la presente investigación.

\section{Autoría}

La primera autora ha participado en la conceptualización, metodología, ha participado en la investigación, en el tratamiento de datos, Simulaciones (Software), análisis formal, visualización. Además, ha escrito el borrador original y ha participado en la revisión y edición. La segunda autora ha participado en la conceptualización, metodología y ha participado en la investigación. Además, en la redacción, revisión, edición y supervisión. El tercer autor ha participado en la conceptualización y metodología. Además, en la revisión y edición. El cuarto autor ha participado en la conceptualización, revisión y edición.

Conflicto de intereses: Los autores declaran que no hay conflicto de intereses. 


\section{Bibliografía}

Attia, S. (2011). A Case Study for a Zero Impact Building in Belgium: Mondo Solar-2002. International Journal of Sustainable Building Technology and Urban Development, 2(2), 137-142. https://doi.org/10.5390/SUSB.2011.2.2.137

Bedoya, C., \& Dzul, L. (2015). Concrete with recycled aggregates as urban sustainability project El concreto con agregados reciclados como proyecto de sostenibilidad urbana, 30, 99-108.

Borucke, M., Moore, D., Cranston, G., Gracey, K., Iha, K., Larson, J., ... Galli, A. (2017). Accounting for demand and supply of the Biosphere' s regenerative capacity: The National Footprint Accounts ' underlying $\quad \ldots \quad$ Ecological Indicators, $\quad$ 24(September), https://doi.org/10.1016/j.ecolind.2012.08.005

Campos Arriaga, L. Estudio de Optimización de la Eficiencia Energética en Viviendas de Interés Social (2011).

Castellano, J., Castellano, D., Ribera, A., \& Ciurana, J. (2015). Developing a simplified methodology to calculate $\mathrm{Co} / \mathrm{m} 2$ emissions per year in the use phase of newly-built, single-family houses. Energy and Buildings, 109(2015), 90-107. https://doi.org/10.1016/j.enbuild.2015.09.038

CENAPRED. (2015). Características e Impacto Socioeconómico de los Principales Desastres Ocurridos en la República Mexicana en el año 2013.

CENAPRED. (2018a). Atlas Nacional de Riesgos. Retrieved November 6, 2018, from http://www.atlasnacionalderiesgos.gob.mx/

CENAPRED. (2018b). Sistema de Consulta de Declaratorias. Retrieved November 6, 2018, from http://www.atlasnacionalderiesgos.gob.mx/archivo/visualizacion-datos.html

Centro de Ciencias de la Atmósfera UNAM. (2013). Atlas Climático Digital de México. Estado de Guerrero. Retrieved November 6, 2018, from http://atlasclimatico.unam.mx/atlas/gro/gro.html

CFE. (2018). Tarifas de consumo eléctrico. Retrieved November 6, 2018, from https://app.cfe.mx/Aplicaciones/CCFE/Tarifas/TarifasCRECasa/Casa.aspx

Chapagain, A., \& Hoekstra, A. (2014). Water footprints of nations - Value of Water Research Report Series No. 16. UNESCO-IHE Institute for Water Education, 1(January 2004). Retrieved from https://www.researchgate.net/publication/200041794_Water_footprints_of_nations_-_Volume_1

Comisión Nacional para el Uso Eficiente de la Energía. (2016a). ANEXO 2. Tablas de probabilidad de presencia de ventiladores en vivienda. In Estudio de Caracterización del Uso de Aire Acondicionado en Viviendas de Interés Social.

Comisión Nacional para el Uso Eficiente de la Energía. (2016b). Estudio de Caracterización del Uso de Aire Acondicionado en Viviendas de Interés Social en México. Retrieved from http://www.gob.mx/conuee/acciones-y-programas/estudio-caracterizacion-del-uso-de-aireacondicionado-en-vivienda-de-interes-social-en-mexico?idiom=es

Comisión Reguladora de Energía. (2017). Registro Nacional de Emisiones (RENE). Retrieved November 6, 2018, from https://www.gob.mx/semarnat/acciones-y-programas/registro-nacional-de-emisionesrene 
CONAGUA. (2018). Tarifas de agua potable y saneamiento en México. Retrieved November 6, 2018, from http://sina.conagua.gob. $\mathrm{mx} /$ sina/tema.php?tema=tarifas\&ver=reporte\&o=0\&n=nacional

CONEVAL. (2016). Índice de Rezago Social 2015 Presentación de resultados. México, D.F. Retrieved from

http://www.coneval.org.mx/Medicion/Documents/Indice_Rezago_Social_2015/Nota_Rezago_Social_2015_vf.pdf Consejo Nacional de Evaluación de la Politica de Desarrollo Social. (2013). Informe de pobreza y evaluación, Guerrero 2012 - 2013. México, D.F.: CONEVAL.

Conticelli, E., Proli, S., \& Tondelli, S. (2017). Integrating energy efficiency and urban densification policies: Two Italian case studies. Energy and Buildings, 155, 308-323. https://doi.org/10.1016/j.enbuild.2017.09.036

Cruz Roja - Media Luna Roja. (2011). El Proyecto Esfera.

Diario oficial de la federación. Lineamientos de operación específicos del fondo de desastres naturales (2011). México: DOF.

Diario Oficial de la Federación. Ley de vivienda (2014). México: DOF.

Diario Oficial de la Federación. PROGRAMA NACIONAL DE VIVIENDA 2014 - 2018, DOF § (2014). Retrieved from http://www.dof.gob.mx/nota_detalle.php?codigo=5342865\&fecha=30/04/2014

Diario Oficial de la Federación. RESOLUCIÓN del H. Consejo de Representantes de la Comisión Nacional de los Salarios Mínimos que fija los salarios mínimos general y profesionales vigentes a partir del 10. de enero de 2018 (2018). México. Retrieved from http://dof.gob.mx/nota_detalle.php?codigo=5508586\&fecha=21/12/2017

Elizondo, A., Pérez-Cirera, V., Strapasson, A., Fernández, J. C., \& Cruz-Cano, D. (2017). Mexico's low carbon futures: An integrated assessment for energy planning and climate change mitigation by 2050. Futures, 93(June), 14-26. https://doi.org/10.1016/j.futures.2017.08.003

FLORES, L. (2015). NORMA MEXICANA NMX-C-404-ONNCCE-2012 PIEZAS PARA USO ESTRUCTURAL. In normas NMX para estructuras de mampostería (Vol. 74, pp. 23-42).

Fuentes Freixanet, V. A. (2009). Modelo de análisis climático y definición de estrategias de diseño bioclimático para diferentes Regiones de la República Mexicana. Universidad Autonoma Metropolitana - Azcapotzalco.

Global Footprint Network National Footprint Accounts. (2018). Factor de equivalencia. Retrieved November 6, 2018, from http://data.footprintnetwork.org

Google maps. (2018). Ubicación Nuevo Azinyahualco. Retrieved from https://www.google.com/maps/place/Azinyahualco,+Gro./@17.3240375,-

99.5628759,1206m/data=!3m1!1e3!4m5!3m4!1s0x85cbbf902998280f:0x57b3b764dc15c6eb!8m2!3d17.4 $062366 ! 4 d-99.5557731$

Ibidhi, R., \& Ben Salem, H. (2018). Water footprint and economic water productivity of sheep meat at farm scale in humid and semi-arid agro-ecological zones. Small Ruminant Research, 166(January), 101-108. https://doi.org/10.1016/j.smallrumres.2018.06.003

INEGI. (2005). Climatología. Retrieved November 6, 2018, from http://www.beta.inegi.org.mx/temas/mapas/climatologia/ 
INEGI. (2010). Población de 5 años y más hablante de lengua indígena por entidad federativa según sexo, $1990 \quad$ a 2010. Retrieved November 6, 2018, from http://www.beta.inegi.org.mx/app/tabulados/pxweb/inicio.html?rxid=75ada3fe-1e52-41b3-bf274cda26e957a7\&db=Lengualndigena\&px=Lengua_1

Jones, P., Li, X. J., Perisoglou, E., \& Patterson, J. (2017). Five energy retrofit houses in South Wales. Energy and Buildings, 154, 335-342. https://doi.org/10.1016/j.enbuild.2017.08.032

Lee, Y. J. (2015). Land, carbon and water footprints in Taiwan. Environmental Impact Assessment Review, 54, 1-8. https://doi.org/10.1016/j.eiar.2015.04.004

Liang, L., Lal, R., Wu, W., Ridoutt, B. G., Du, Z., Li, L., ... Zhao, G. (2018). The water footprint and validity analysis of ecological engineering in North Beijing, China. Journal of Cleaner Production, 172, 18991909. https://doi.org/10.1016/j.jclepro.2017.11.251

Mac Donald, J. (1987). Vivienda Progresiva. Corporación de Promoción Universitaria. Santiago, Chile

Martínez Rocamora, A., Solís-Guzmán, J., \& Marrero, M. (2017). Ecological footprint of the use and maintenance phase of buildings: Maintenance tasks and final results. Energy and Buildings, 155, 339351. https://doi.org/10.1016/j.enbuild.2017.09.038

Moran, F., Blight, T., Natarajan, S., \& Shea, A. (2014). The use of Passive House Planning Package to reduce energy use and CO2emissions in historic dwellings. Energy and Buildings, 75, 216-227. https://doi.org/10.1016/j.enbuild.2013.12.043

Naciones Unidas - Derechos Humanos - ONU HABITAT. (1948). El derecho a una vivienda adecuada. Derechos Humanos, 21(1), 3-10. https://doi.org/10.1017/CBO9781107415324.004

Neuman, M. (2014). The Compact City Fallacy, 11-26. https://doi.org/10.1177/0739456X04270466

Pardo, N., \& Thiel, C. (2012). Evaluation of several measures to improve the energy efficiency and CO2emission in the European single-family houses. Energy and Buildings, 49, 619-630. https://doi.org/10.1016/j.enbuild.2012.03.006

Pfister, S., Boulay, A. M., Berger, M., Hadjikakou, M., Motoshita, M., Hess, T., ... Henderson, A. (2017). Understanding the LCA and ISO water footprint: A response to Hoekstra (2016) "A critique on the water-scarcity weighted water footprint in LCA." Ecological Indicators, 72, 352-359. https://doi.org/10.1016/j.ecolind.2016.07.051

PROFEPA. (2015). Clausura de obra Nuevo Azinyahualco. Chilpancingo, Guerrero. Retrieved from https://www.profepa.gob.mx/innovaportal/v/7629/1/mx/clausura_profepa_construccion_ilegal_en_te rrenos_forestales_de_chilpancingo_guerrero.html

R. Džiugait 'e-Tum 'enien 'e, V. Jankauskas, V. M. 'e. (2015). Energy Balance of Low Energy House. JOULNAL OF CIVIL ENGINEERING AND MANAGEMENT, (December). https://doi.org/10.1177/096228029400300203

Renato D’Alençon, Catalina Justiniano, Francisca Márquez, C. V. (2008). Parámetros y estándares de habitabilidad: calidad en la vivienda, el entorno inmediato y el conjunto habitacional. In Camino al bicentenario, propuestas para Chile. (pp. 271-304). 
Roibás, L., Cuevas, A., Vázquez, M. E., Vilas, M., \& Hospido, A. (2018). Using water scarcity footprint to choose the most suitable location for forest carbon sinks: A case study. Sustainable Production and Consumption, 16, 1-12. https://doi.org/10.1016/j.spc.2018.06.001

SAINZ, M., \& DE TORRES, M. Catalogo de productos Fetdeterra.

Sarmiento, P., \& Hormazábal, N. (2016). Habitabilidad térmica en las viviendas básicas de la Zona central de chile, a la luz de los resultados preliminares del proyecto FONDE FD001103941, 1-8.

SEMARNAT/CONAVI. (2012). NAMA. ACCIONES NACIONALES APROPIADAS DE MITIGACIÓN. Ciudad de México, México. Retrieved from www.conavi.gob.mx/viviendasustentable\%0A

Servicio Metereológico Nacional. (2010). Normales Climatologicas. Retrieved November 6, 2018, from http://smn.cna.gob.mx/es/informacion-climatologica-ver-estado?estado=gro

Su, Y., Gao, W., Guan, D., \& Su, W. (2018). Dynamic assessment and forecast of urban water ecological footprint based on exponential smoothing analysis. Journal of Cleaner Production, 195, 354-364. https://doi.org/10.1016/j.jclepro.2018.05.184

Szokolay, S. (2004). Introduction to architectural sciences the basis of sustainable design. Amsterdam: Elsevier.

The World Bank and The United Nations. (2010). Natural hazards, unnatural disasters the economics of effective prevention. https://doi.org/10.1596/978-0-8213-8050-5

Valančius, K., Vilutienè, T., \& Rogoža, A. (2018). Analysis of the payback of primary energy and CO2emissions in relation to the increase of thermal resistance of a building. Energy and Buildings, 179, 39-48. https://doi.org/10.1016/j.enbuild.2018.08.037

Zhang, G., Setunge, S., \& van Elmpt, S. (2014). Using Shipping Containers to Provide Temporary Housing in Post-disaster Recovery: Social Case Studies. Procedia Economics and Finance, 18(September), 618625. https://doi.org/10.1016/S2212-5671(14)00983-6

Zhang, J., Xie, Y., Luan, B., \& Chen, X. (2015). Urban macro-level impact factors on Direct CO2 Emissions of urban residents in China. Energy and Buildings, 107, 131-143. https://doi.org/10.1016/j.enbuild.2015.08.011 